\title{
THE CARNEGIE SUPERNOVA PROJECT: SECOND PHOTOMETRY DATA RELEASE OF LOW-REDSHIFT TYPE Ia SUPERNOVAE
}

\author{
Maximilian D. Stritzinger ${ }^{1,2,3}$, M. M. Phillips ${ }^{3}$, Luis N. Boldi ${ }^{4}$, Chris Burns $^{5}$, Abdo Campillay ${ }^{3}$, \\ Carlos Contreras 6 , Sergio Gonzalez ${ }^{7}$, Gastón Folatelli ${ }^{8}$, Nidia Morrell ${ }^{3}$, Wojtek Krzeminski ${ }^{3}$, \\ Miguel Roth ${ }^{3}$, Francisco Salgado ${ }^{9}$, D. L. DePoy ${ }^{10}$, Mario Hamuy ${ }^{11}$, Wendy L. Freedman ${ }^{5}$, \\ Barry F. Madore ${ }^{5,12}$, J. L. Marshall ${ }^{10}$, Sven E. Persson ${ }^{5}$, Jean-Philippe Rheault ${ }^{10}$, \\ Nicholas B. SuntzefF ${ }^{10}$, Steven Villanueva ${ }^{10}$, Weidong Li ${ }^{13}$, and Alexei V. Filippenko ${ }^{13}$ \\ ${ }^{1}$ The Oskar Klein Centre, Department of Astronomy, Stockholm University, AlbaNova, 10691 Stockholm, Sweden; max.stritzinger@astro.su.se \\ ${ }^{2}$ Dark Cosmology Centre, Niels Bohr Institute, University of Copenhagen, 2100 Copenhagen Ø, Denmark; max @ dark-cosmology.dk \\ ${ }^{3}$ Carnegie Observatories, Las Campanas Observatory, La Serena, Chile; mstritzinger@1co.cl \\ ${ }^{4}$ Argelander Institut für Astronomie, Universität Bonn, D-53111 Bonn, Germany \\ ${ }^{5}$ Observatories of the Carnegie Institution for Science, Pasadena, CA 91101, USA \\ ${ }^{6}$ Centre for Astrophysics \& Supercomputing, Swinburne University of Technology, Victoria 3122, Australia \\ ${ }^{7}$ Atacama Large Millimeter/Submillimeter Array, European Southern Observatory, Chile \\ ${ }^{8}$ Institute for the Physics and Mathematics of the Universe (IPMU), University of Tokyo, 5-1-5 Kashiwanoha, Kashiwa, Chiba 277-8583, Japan \\ ${ }^{9}$ Leiden Observatory, Leiden University, NL-2300 RA Leiden, The Netherlands \\ ${ }^{10}$ George P. and Cynthia Woods Mitchell Institute for Fundamental Physics and Astronomy, Department of Physics and Astronomy, \\ Texas A\&M University, College Station, TX 77843, USA \\ ${ }^{11}$ Departamento de Astronomía, Universidad de Chile, Santiago, Chile \\ ${ }^{12}$ Infrared Processing and Analysis Center, Caltech/Jet Propulsion Laboratory, Pasadena, CA 91125, USA \\ ${ }^{13}$ Department of Astronomy, University of California, Berkeley, CA 94720-3411, USA \\ Received 2011 May 23; accepted 2011 August 18; published 2011 October 3
}

\begin{abstract}
The Carnegie Supernova Project (CSP) was a five-year observational survey conducted at Las Campanas Observatory that obtained, among other things, high-quality light curves of $\sim 100$ low-redshift Type Ia supernovae (SNe Ia). Presented here is the second data release of nearby SN Ia photometry consisting of 50 objects, with a subset of 45 having near-infrared follow-up observations. Thirty-three objects have optical pre-maximum coverage with a subset of 15 beginning at least five days before maximum light. In the near-infrared, 27 objects have coverage beginning before the epoch of $B$-band maximum, with a subset of 13 beginning at least five days before maximum. In addition, we present results of a photometric calibration program to measure the CSP optical ( $u B g V r i)$ bandpasses with an accuracy of $\sim 1 \%$. Finally, we report the discovery of a second SN Ia, SN 2006ot, similar in its characteristics to the peculiar SN 2006bt.
\end{abstract}

Key words: galaxies: distances and redshifts - supernovae: general

Online-only material: color figures, extended figure, machine-readable and VO tables

\section{INTRODUCTION}

The current cosmological paradigm ( $\Lambda$ cold dark matter) suggests that the universe is composed of $\sim 4.4 \%$ baryonic matter, $\sim 21.3 \%$ cold dark matter, and $\sim 74.2 \%$ dark energy (e.g., Dunkley et al. 2009). The luminosity distances of Type Ia supernovae ( $\mathrm{SNe}$ Ia) provided the first strong evidence that the expansion rate of the universe is currently accelerating (Riess et al. 1998; Perlmutter et al. 1999), and that dark energy having negative pressure is the major contributor to the overall energy budget of the universe. These findings have been further substantiated through the detection of the late-time-integrated Sachs-Wolfe effect (Giannantonio et al. 2008) and X-ray cluster distances (e.g., Allen et al. 2007).

To date, much effort and resources have been directed toward characterizing the nature of dark energy, resulting in more than $1000 \mathrm{SNe}$ Ia being observed, extending from the smooth Hubble flow out to a redshift $(z)$ of 1.7. The current data have revealed that the equation-of-state parameter of the dark energy, $w=P /\left(\rho c^{2}\right)$, is consistent with a cosmological constant, $w=-1$ (Astier et al. 2006; Wood-Vasey et al. 2007; Riess et al. 2007; Freedman et al. 2009; Sullivan et al. 2011). Advancements in our understanding of dark energy will come from future experiments that seek to measure the time derivative of $w$. The success and credibility of these experiments will require percent-level accuracy in SN distances, something that has yet to be demonstrated. To achieve this will require both a deeper theoretical understanding of the progenitors and explosion mechanism as well as, on the observational side, a significant sample of well-calibrated low- $z$ SNe Ia monitored over a wide wavelength range.

The Carnegie Supernova Project (CSP) is the umbrella designation of a five-year (2004-2009) National Science Foundation (NSF) funded observational campaign consisting of low- and high $z$ components. The overriding goal of the low $z$ component was to construct an atlas of $\sim 100$ high signal-to-noise ratio $(\mathrm{S} / \mathrm{N}) \mathrm{SN}$ Ia light curves in a homogeneous and welldefined photometric system in the optical and near-infrared (IR). These data will serve as a fundamental reference to anchor future Hubble diagrams that will contain thousands of high- $z$ $\mathrm{SNe}$ Ia observed by future all-sky surveys. The objective of the high- $z$ portion of the CSP was to construct a rest-frame $i$-band Hubble diagram in order to minimize the effects of dust reddening. At the end of the latter observing program, $71 \mathrm{SNe}$ Ia were observed at cosmological distances out to $z \approx 0.7$. A subset of these objects was combined with our initial low- $z$ sample to construct the Hubble diagram presented by Freedman et al. (2009).

By the end of the fifth and final year (2009 June), the low- $z$ part of the CSP had obtained photometric follow-up observations of $129 \mathrm{SNe}$ Ia. In the first of a series of papers, 
we presented optical $(u B g V r i)$ and near-IR $\left(Y J H K_{s}\right)$ light curves of $35 \mathrm{SNe}$ Ia (Contreras et al. 2010), along with a detailed analysis (Folatelli et al. 2010). A highlight of the Folatelli et al. study is the discrepancy found between the value of the reddening parameter, $R_{V}$, as determined through the comparison of color excesses to the method of leaving $R_{V}$ as a free parameter and minimizing the dispersion in the Hubble diagram. When excluding the two most highly reddened objects from the first sample, a comparison of colors or color excesses suggests agreement with the canonical Milky Way value, $R_{V} \approx 3.0$. However, when one minimizes the dispersion in the Hubble diagram with $R_{V}$ treated as a free parameter, lower values of $R_{V} \approx 1-2$ are obtained. This discrepancy implies that there is an intrinsic color variation of SNe Ia that correlates with luminosity, but is independent of the light-curve decline-rate parameter $\Delta m_{15} \cdot{ }^{14}$ To address this apparent inconsistency demands an expanded sample of well-observed multi-band SNe Ia.

In this paper, we present the second CSP release of SN Ia light curves. The sample consists of 50 objects observed between 2005 and 2009, with a subset of 45 objects having accompanying near-IR observations. The light curves presented here are a significant addition to a growing sample of low- $z$ SNe Ia that have been observed in well-understood and stable photometric systems (Wood-Vasey et al. 2008; Hicken et al. 2009; Contreras et al. 2010; Ganeshalingam et al. 2010). A third and final paper containing optical and near-IR light curves of an additional $\sim 20-25$ objects will complete the full data release of low $-z$ SNe Ia observed by the CSP.

Additional aims of the CSP included the construction of substantial samples of Type II SNe $(\sim 100)$ and Type Ib/c $\mathrm{SNe}(\sim 25)$. By the end of our observational program, these goals were successfully achieved, and a majority of the data are currently being analyzed. The CSP was not limited to only photometric follow-up observations; a notable commitment was also made to obtain contemporaneous optical spectroscopy. In a companion paper currently in preparation an analysis of spectrophotometry of $\sim 80 \mathrm{SNe}$ Ia monitored by the CSP will be presented. Additionally, a second analysis paper combining the CSP's first 85 published low- $z$ SNe Ia will be presented in the near future.

The organization of this paper is as follows. Section 2 briefly describes the observations, Section 3 discusses results obtained from a calibration program to measure the optical and near-IR transmission functions of the Henrietta Swope $1 \mathrm{~m}$ and Irénée du Pont $2.5 \mathrm{~m}$ telescopes equipped with CSP filters, Section 4 presents the final light curves, Section 5 compares two peculiar objects, and Section 6 contains our conclusions.

\section{OBSERVATIONS}

A complete overview of the CSP including the goals of the project, the facilities at Las Campanas Observatory (LCO) used to conduct photometric and spectroscopic data, and the observing procedures are given by Hamuy et al. (2006). A detailed and up-to-date description of the data-reduction procedures, the host-galaxy subtraction techniques, and computation of definitive photometry in the natural system of the LCO facilities is given in the first data release of SN Ia photometry (Contreras et al. 2010). It is not our intention to repeat the material contained

\footnotetext{
14 The decline-rate parameter is traditionally defined as the difference in magnitude of the $B$-band light curve from peak brightness to 15 days later. The value of $\Delta m_{15}$ is correlated with absolute peak magnitude in such a way that low-luminosity SNe Ia exhibit a faster decline rate (Phillips 1993).
}

in these previous publications, so in this section we provide only a brief summary of our methodology.

The CSP was a follow-up program of southern and equatorial $\mathrm{SNe}$ discovered by various sources ranging from dedicated backyard amateurs to professional searches. Five observational campaigns, each nine months in duration, were conducted between 2004 and 2009. The primary criteria used to select an object for monitoring were (1) that it was caught young, preferably before maximum light, or within a few weeks after explosion in the case of Type II plateau objects, and (2) that the peak magnitude was $\sim 18$ or brighter. The workhorse of the CSP was the Henrietta Swope $1 \mathrm{~m}$ telescope equipped with both a direct imaging CCD camera that we refer to as SITe3 and a near-IR imager called RetroCam. Additional nearIR imaging and limited optical observations were obtained with the du Pont $2.5 \mathrm{~m}$ telescope equipped with the Wide Field IR Camera (WIRC) and a direct optical imager referred to as Tek 5. Typically a year or more after the last observation of any given SN, deep "template" images of its host galaxy were obtained with the du Pont telescope. These high $\mathrm{S} / \mathrm{N}$ templates are used to subtract away the host-galaxy light at the position of the SN in each science image. This procedure is essential for obtaining precise photometry, particularly if the SN occurred in the inner region of the host galaxy or in a spiral arm.

Template subtraction has been performed on the science images of all objects presented here except SN 2007if (nearIR) and SN 2009dc (optical and near-IR). As these two events are super-Chandrasekhar candidates, we recognize the interests within the community to promptly make their photometry public. Given the position of both objects in their respective host galaxies, we suspect that the photometry suffers negligible background contamination. Nevertheless, in the near future templates of these objects will be acquired, and the subsequent template-subtracted photometry will be available on the CSP Web site.

Photometry of each $\mathrm{SN}$ is computed differentially with respect to a local sequence of stars. Absolute photometry of each local sequence is determined relative to photometric standard stars which were observed over a minimum of three photometric nights. The master list of optical photometric standard stars used to calibrate the local sequences is composed of stars in common with the Landolt (1992) and Smith et al. (2002) catalogs. In the near-IR, absolute photometry of each local sequence is computed relative to Persson et al. (1998) standards.

The CSP has elected to publish photometry on the natural system of the Swope and du Pont telescopes. The reasons for this are highlighted by Contreras et al. (2010), and boil down to providing the photometry in a form that simplifies the process of combining it with data sets obtained by other groups. Armed with spectral templates and well-defined systemresponse functions, CSP photometry can be transformed or "S-corrected" (Stritzinger et al. 2002) to any desired userdefined photometric system in a straightforward manner.

The SNe contained in this data release were observed from mid-2005 to early 2009. A mosaic consisting of a $V$-band image of each $\mathrm{SN}$ is presented in Figure 1. General properties related to each object are provided in Table 1 . Specifically, this includes (1) the coordinates, (2) identification, morphology, and redshift of the host galaxy, (3) a reference to the IAUC or CBET reporting the discovery, and (4) the name of the discovery individual(s) or group(s).

Basic photometric and spectroscopic information on each object is compiled in Table 2, consisting of (1) an estimate of 


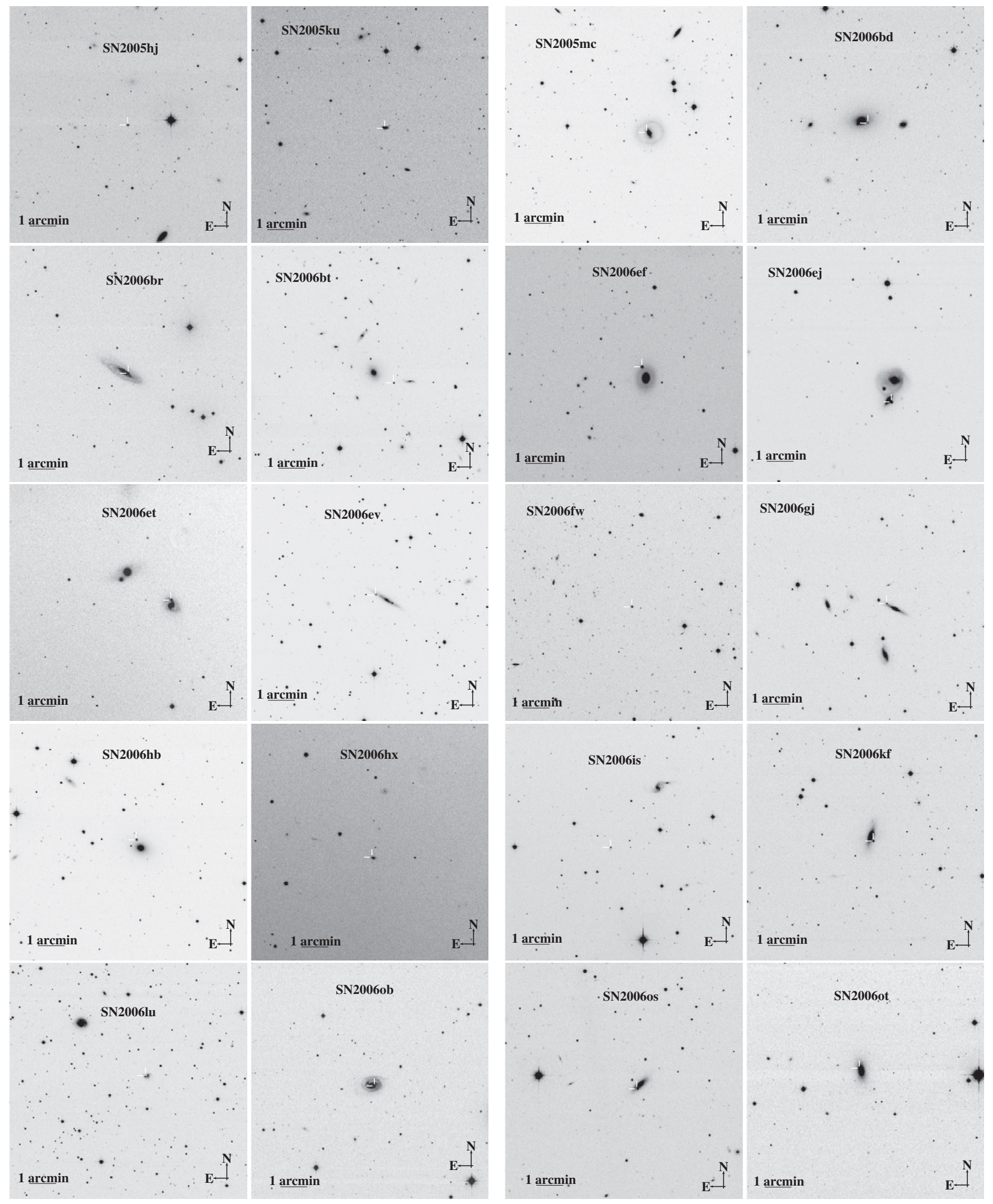

Figure 1. Mosaic of $V$-band CCD images of $50 \mathrm{SNe}$ Ia observed by the CSP.

the decline-rate parameter, $\Delta m_{15}$ (see Section 4 for details), (2) the number of nights each SN was observed, (3) the number of nights that standard-star fields were observed under photometric conditions, and (4) the $\mathrm{SN}$ spectral subtype. Values of $\Delta m_{15}$ were computed using the SNooPy Python package (SNooPy; Burns et al. 2011), while the SuperNova IDentification code (Blondin \& Tonry 2007) provided aid in determining spectral subtypes. Finally, the last column of Table 2 lists the epoch of the spectrum 


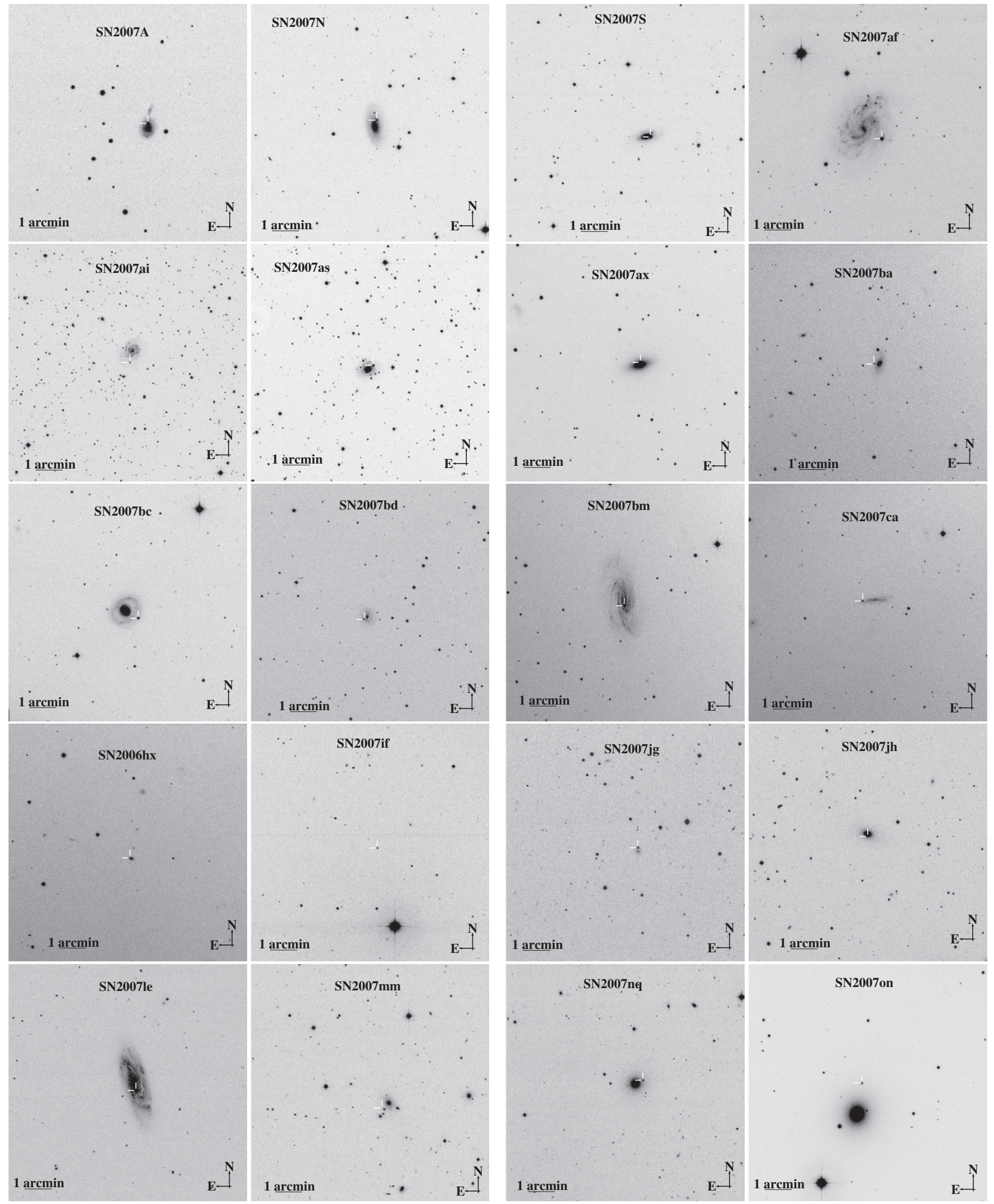

Figure 1. (Continued)

(relative to the time of $B$-band maximum) used to determine the spectral subtype.

Examination of the light-curve properties and optical spectra reveals that 36 of the objects in this data release are normal
SNe Ia, 8 are of the low-luminosity SN 1986G (Phillips et al. 1987) or SN 1991bg (Filippenko et al. 1992a; Leibundgut et al. 1993) variety, 2 are of the slow-declining SN 1991T-like subtype (Filippenko et al. 1992b; Phillips et al. 1992), 2 are considered 

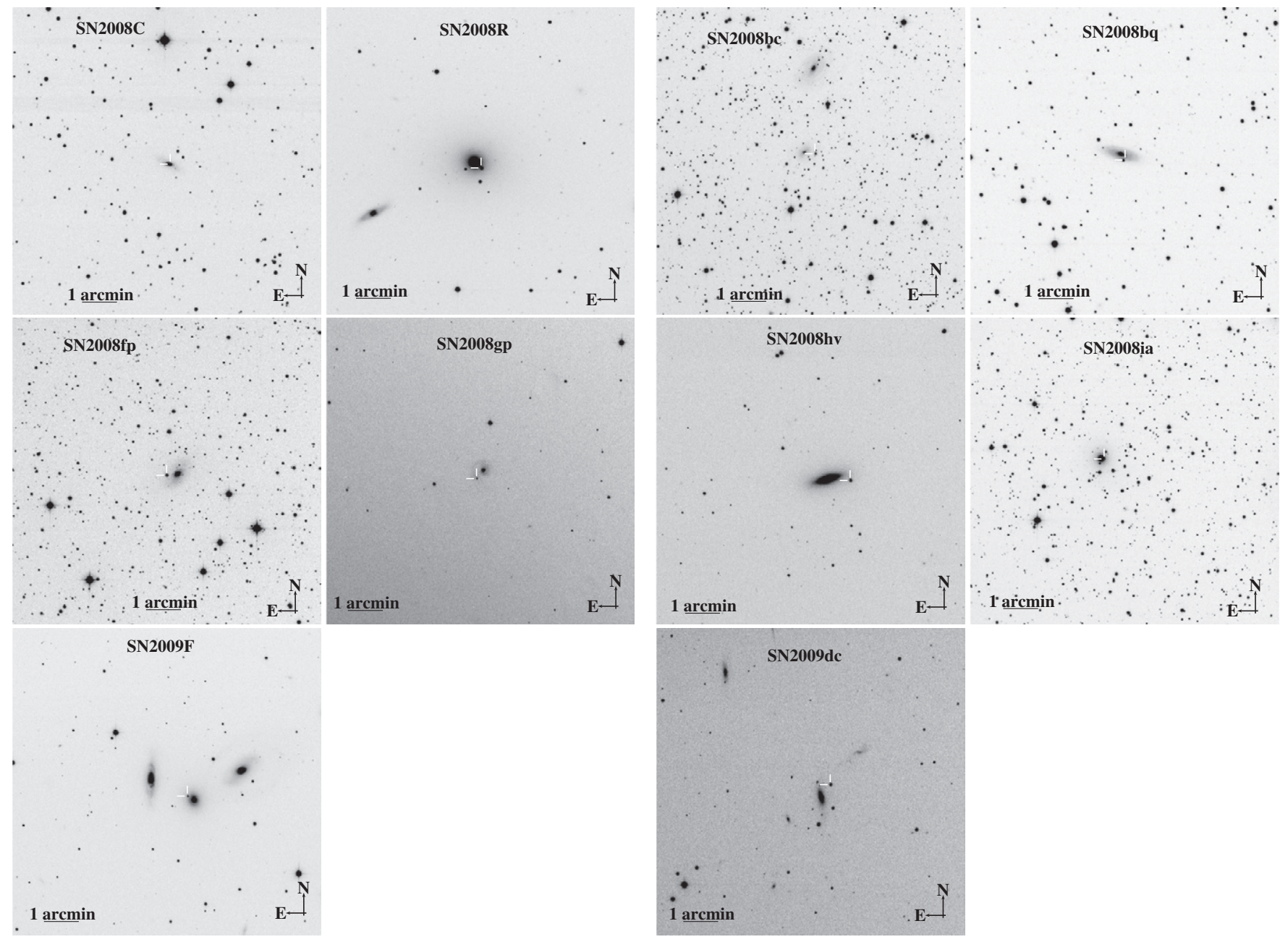

Figure 1. (Continued)

possible super-Chandrasekhar SNe (e.g., see Howell et al. 2006), and 2 objects, SN 2006bt and SN 2006ot, are uniquely peculiar (Foley et al. 2010).

Optical and near-IR photometry of each local sequence in the standard Landolt ( $B V$; Landolt 1992), Smith $\left(u^{\prime} g^{\prime} r^{\prime} i^{\prime}\right.$; Smith et al. 2002), and Persson ( $J H K_{s}$; Persson et al. 1998) photometric systems are given in Table 3 . The $Y$-band photometry is calibrated relative to the $\left(Y-K_{s}\right)$ and $\left(J-K_{s}\right)$ color relation given by Hamuy et al. (2006, see Appendix C, Equation (C2)). The quoted uncertainty accompanying the magnitudes of each star corresponds to the weighted average of the instrumental errors computed from several measurements obtained over the course of multiple photometric nights (see Columns 5 and 6 of Table 2).

\section{CSP BANDPASSES}

In the past, photometry of local SNe Ia has often been obtained with poorly quantified system (telescope + instrument + filters) response functions. This problem makes it difficult to precisely combine low- and high- $z$ SN Ia samples, and contributes to the systematic error budget of cosmological parameters. To maximize the potential of our low- $z$ sample, we have devised a calibration program to measure with $1 \%$ accuracy the response of the CSP bandpasses on the Swope (optical + near-IR) and du Pont (near-IR) telescopes. In this section, we describe the photometric calibration program and present the scanned optical response functions. The near-IR scanned response functions will be presented in a forthcoming data paper. A more complete discussion of the technical aspects of this experiment, including a schematic of the setup, is presented by Rheault et al. (2010).

\subsection{Experimental Setup}

The experimental setup consists of a broadband light source that illuminates a monochromator. Two light sources provide sufficient illumination in the optical and near-IR regimes. In the optical, a $75 \mathrm{~W}$ Xenon lamp placed in an ellipsoidal reflector gives a smooth continuum from $3000 \AA$ to $8000 \AA$, while redward from $8000 \AA$ to $24000 \AA$ a 100 W quartz tungsten halogen lamp is used. An optimized setup of collimating optics directs the light to the entrance slit of a monochromator. The collimating optics used for projection consist of UV grade fused silica. The monochromator selects a narrow bandwidth of light (between 20 and $50 \AA$ ) that is fed into a fiber bundle made of 10 individual $600 \mu \mathrm{m}$ diameter fibers arranged as a vertical line at the exit slit of the monochromator, while at the end of the fiber line they are arranged as a compact spot. The custom fiber bundle was manufactured by Fibertech Optical using $600 \mu \mathrm{m}$ fibers from Polymicro (model FBP). The fibers are made of a special broadband glass that has high transmission from $3500 \AA$ up to $18000 \AA$. Outside of this wavelength range, the fiber transmission begins to drop, but there is still enough power to measure the near-IR throughput accurately. The output of the 
Table 1

General Properties of $50 \mathrm{SNe}$ Ia

\begin{tabular}{|c|c|c|c|c|c|c|c|}
\hline $\mathrm{SN}$ & $\begin{array}{c}\mathrm{SN} \\
\alpha(2000)\end{array}$ & $\begin{array}{c}\mathrm{SN} \\
\delta(2000)\end{array}$ & $\begin{array}{c}\text { Host } \\
\text { Galaxy }\end{array}$ & Morphology $\mathrm{y}^{\mathrm{a}}$ & $z_{\text {helio }}{ }^{a}$ & $\begin{array}{l}\text { Discovery } \\
\text { Reference }\end{array}$ & Discovery Individual/Group ${ }^{b}$ \\
\hline 2005hj & $01: 26: 48.27$ & $-01: 14: 16.8$ & В012415.21 - 012950.3 & $\ldots$ & $0.0580^{\dagger}$ & CBET 266 & ROTSE-III \\
\hline $2005 \mathrm{ku}$ & $22: 59: 42.61$ & $+00: 00: 49.3$ & 2MASX J22594265 - 0000478 & $\ldots$ & $0.0454^{\dagger}$ & CBET 304 & SDSS-II \\
\hline $2005 \mathrm{mc}$ & $08: 27: 06.36$ & $+21: 38: 45.6$ & UGC 04414 & SOa & 0.0252 & CBET 331 & THCA \\
\hline 2006bd & $11: 38: 28.46$ & $+20: 31: 34.4$ & UGC 6609 & $\mathrm{E}$ & 0.0257 & CBET 448 & Puckett \\
\hline $2006 \mathrm{br}$ & $13: 30: 01.80$ & $+13: 24: 56.8$ & NGC 5185 & $\mathrm{Sb}$ & 0.0246 & CBET 482 & Puckett \\
\hline $2006 \mathrm{bt}$ & $15: 56: 30.53$ & $+20: 02: 45.3$ & CGCG $108-013$ & $\mathrm{SA} 0 / \mathrm{a}$ & 0.0322 & CBET 485 & LOSS \\
\hline 2006ef & 02:04:19.51 & $-08: 43: 42.2$ & NGC 809 & (R)S0 & 0.0179 & CBET 597 & LOSS \\
\hline 2006ej & $00: 38: 59.77$ & -090056.6 & NGC 191A & So pec sp & 0.0205 & CBET 603 & LOSS \\
\hline 2006et & $00: 42: 45.82$ & $-23: 33: 30.4$ & NGC 232 & SB(r)a? pec & 0.0226 & CBET 616 & Itagaki \\
\hline $2006 \mathrm{ev}$ & $21: 30: 59.26$ & $+13: 59: 21.2$ & UGC 11758 & $\mathrm{Sbc}$ & 0.0287 & IAUC 8747 & Ory \\
\hline $2006 f w$ & $01: 47: 10.34$ & $-00: 08: 49.2$ & anonymous & & $0.0835^{\ddagger}$ & CBET 627 & SDSS-II \\
\hline $2006 \mathrm{gj}$ & $03: 17: 35.80$ & $-01: 41: 30.2$ & UGC 2650 & $\mathrm{Sab}$ & $0.0284^{\dagger}$ & CBET 631 & Puckett \\
\hline $2006 \mathrm{hb}$ & 05:02:01.28 & $-21: 07: 55.1$ & MCG-04 - $12-34$ & $\mathrm{E} ?$ & 0.0153 & CBET 649 & LOSS \\
\hline $2006 h x$ & $01: 13: 57.31$ & $+00: 22: 18.0$ & 2MASX J01135716 + 0022171 & So & 0.0455 & CBET 656 & SDSS-II \\
\hline 2006is & $05: 17: 34.37$ & $-23: 46: 54.2$ & В051529.79- 235009.8 & $\ldots$ & $0.0314^{\dagger}$ & CBET 659 & LOSS \\
\hline $2006 \mathrm{kf}$ & $03: 41: 50.48$ & $+08: 09: 25.0$ & UGC 2829 & S0 & 0.0213 & CBET 686 & LOSS \\
\hline 2006lu & $09: 15: 17.63$ & $-25: 36: 00.3$ & anonymous & $\ldots$ & $0.0534^{\dagger}$ & IAUC 8770 & LOSS \\
\hline $2006 \mathrm{ob}$ & $01: 51: 48.11$ & $+00: 15: 48.3$ & UGC 1333 & $\mathrm{Sb}$ & 0.0592 & CBET 745 & LOSS/SDSS-II \\
\hline 2006 os & 02:55:01.10 & $+16: 00: 34.8$ & UGC 2384 & $\mathrm{~S}$ & 0.0328 & IAUC 8779 & ROTSE-III/LOSS \\
\hline 2006 ot & $02: 15: 04.84$ & $-20: 45: 58.2$ & ESO 544-G31 & $\mathrm{Sa}$ & 0.0531 & IAUC 8779 & Puckett/LOSS \\
\hline 2007A & $00: 25: 16.66$ & $+12: 53: 12.5$ & NGC 105 & $\mathrm{Sab}$ & 0.0177 & CBET 795 & Puckett/LOSS \\
\hline $2007 \mathrm{~N}$ & $12: 49: 01.25$ & $-09: 27: 10.2$ & $\mathrm{MCG}-01-33-012$ & $\mathrm{SA}(\mathrm{s}) \mathrm{a}$ & 0.0129 & CBET 818 & LOSS \\
\hline $2007 \mathrm{~S}$ & $10: 00: 31.26$ & $+04: 24: 26.2$ & UGC 5378 & $\mathrm{Sb}$ & 0.0139 & CBET 825 & Puckett \\
\hline 2007 af & $14: 22: 21.03$ & $-00: 23: 37.6$ & NGC 5584 & $\mathrm{SAB}(\mathrm{rs}) \mathrm{cd}$ & 0.0055 & CBET 863 & Itagaki \\
\hline 2007ai & $16: 12: 53.74$ & $-21: 37: 48.7$ & MCG-04-38-004 & $\mathrm{Sc}$ & 0.0317 & CBET 870 & LOSS \\
\hline 2007 as & $09: 27: 36.01$ & $-80: 10: 39.2$ & ESO $18-\mathrm{G} 18$ & $\mathrm{SB}(\mathrm{rs}) \mathrm{c}$ & 0.0176 & CBET 888 & Tengra-II \\
\hline $2007 a x$ & $08: 22: 43.26$ & $+22: 33: 16.9$ & NGC 2577 & So & 0.0069 & CBET 904 & Arbour/Itagaki \\
\hline $2007 \mathrm{ba}$ & $15: 16: 42.63$ & $+07: 23: 47.8$ & UGC 9798 & $\mathrm{~S} 0 / \mathrm{a}$ & 0.0385 & CBET 911 & LOSS \\
\hline $2007 b c$ & $11: 19: 14.57$ & $+20: 48: 32.5$ & UGC 6332 & (R)SBa & 0.0208 & CBET 913 & LOSS \\
\hline $2007 \mathrm{bd}$ & $08: 31: 33.28$ & $-01: 11: 58.0$ & UGC 4455 & $\mathrm{SB}(\mathrm{r}) \mathrm{a}$ & 0.0309 & CBET 914 & LOSS \\
\hline $2007 \mathrm{bm}$ & $11: 25: 02.30$ & $-09: 47: 53.8$ & NGC 3672 & $\mathrm{SA}(\mathrm{s}) \mathrm{c}$ & 0.0062 & CBET 936 & Perth \\
\hline $2007 \mathrm{ca}$ & $13: 31: 05.81$ & $-15: 06: 06.6$ & MCG-02-34-61 & Sc pec sp & 0.0141 & CBET 945 & LOSS \\
\hline $2007 h x$ & 02:06:27.08 & $-00: 53: 58.3$ & SDSS J020627.93 - 005353.0 & $\ldots$ & 0.0800 & CBET 1057 & SDSS-II \\
\hline 2007if & $01: 10: 51.37$ & $+15: 27: 39.93$ & anonymous & $\ldots$ & $0.0742^{\mp}$ & CBET 1059 & ROTSE-IIIb/SN Factory \\
\hline 2007jg & $03: 29: 50.82$ & $+00: 03: 24.6$ & SDSS J032950.83 + 000316.0 & $\ldots$ & 0.0371 & CBET 1076 & SDSS-II \\
\hline $2007 \mathrm{jh}$ & $03: 36: 01.54$ & $+01: 06: 12.2$ & CGCG $391-014$ & $\ldots$ & 0.0408 & CBET 1076 & SDSS-II \\
\hline 2007le & $23: 38: 48.41$ & $-06: 31: 21.3$ & NGC 7721 & $\mathrm{SA}(\mathrm{s}) \mathrm{c}$ & 0.0067 & CBET 1100 & Monard \\
\hline $2007 \mathrm{~mm}$ & $01: 05: 46.67$ & $-00: 45: 31.8$ & SHK 035 & $\ldots$ & 0.0665 & CBET 1102 & SDSS-II \\
\hline $2007 \mathrm{nq}$ & $00: 57: 33.57$ & $-01: 23: 19.0$ & UGC 595 & $\mathrm{E}$ & 0.0450 & CBET 1106 & ROTSE-IIIb \\
\hline 2007on & $03: 38: 50.90$ & $-35: 34: 30.0$ & NGC 1404 & E1 & 0.0065 & CBET 1121 & TAROT \\
\hline $2008 \mathrm{C}$ & $06: 57: 11.53$ & $+20: 26: 13.7$ & UGC 3611 & $\mathrm{~S} 0 / \mathrm{a}$ & 0.0166 & CBET 1195 & Puckett \\
\hline $2008 \mathrm{R}$ & 03:03:53.70 & $-11: 59: 39.4$ & NGC 1200 & $\mathrm{SA}(\mathrm{s}) 0$ & 0.0135 & CBET 1230 & Itagaki \\
\hline $2008 b c$ & $09: 38: 31.23$ & $-63: 58: 25.6$ & KK 1524 & $\mathrm{~S}$ & 0.0151 & CBET 1301 & CHASE \\
\hline $2008 \mathrm{bq}$ & $06: 41: 02.51$ & $-38: 02: 19.0$ & ESO $308-G 25$ & $\mathrm{Sa}$ & 0.0340 & CBET 1328 & Tengra-II \\
\hline $2008 f p$ & $07: 16: 32.60$ & $-29: 19: 31.8$ & ESO 428-G014 & $\mathrm{SAB}(\mathrm{r}) 0$ pec & 0.0057 & CBET 1506 & CHASE \\
\hline $2008 \mathrm{gp}$ & $03: 23: 00.73$ & $+01: 21: 42.8$ & $\mathrm{MCG}+00-9-74$ & $(\mathrm{R}) \mathrm{SAB}(\mathrm{r}) \mathrm{a}$ & 0.0334 & CBET 1555 & LOSS \\
\hline 2008hv & 09:07:34.06 & $+03: 23: 32.1$ & NGC 2765 & S0 & 0.0126 & CBET 1601 & CHASE \\
\hline $2008 \mathrm{ia}$ & $08: 50: 35.15$ & $-61: 16: 40.6$ & ESO $125-6$ & So & 0.0217 & CBET 1612 & CHASE \\
\hline $2009 \mathrm{~F}$ & $04: 59: 23.56$ & $-11: 07: 50.1$ & NGC 1725 & So & 0.0130 & CBET 1650 & CHASE \\
\hline $2009 \mathrm{dc}$ & $15: 51: 12.12$ & $+25: 42: 28.0$ & UGC 10064 & So & 0.0214 & CBET 1762 & Puckett \\
\hline
\end{tabular}

Notes.

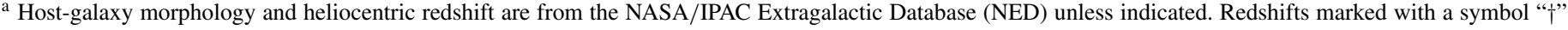
were computed via host spectra obtained by the CSP, while " $\ddagger$ ” and “ $\mp ”$ are from the SDSS-II SN survey and Scalzo et al. (2010), respectively.

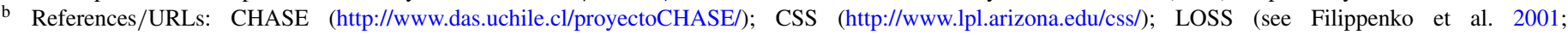

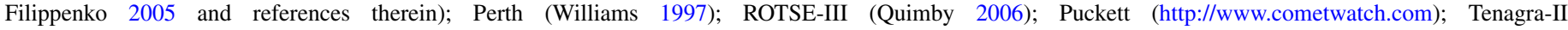
(http://www.tenagraobservatories.com/Discoveries.htm); SDSS-II (Frieman et al. 2008).

fiber bundle is projected onto a highly reflective flat-field screen using an Engineered Diffuser from RPC Photonics (model EDC40) that ensures a uniform distribution of the light. The glass optics of the diffuser have a polymer coating to enhance the $\mathrm{UV}$ transmission. One of the fibers is routed to an MS125 spectrometer from Newport, which measures in real time the central wavelength and the FWHM intensity of the illuminating source to within an accuracy of $1 \AA$. The flat-field screen is made of lightweight honeycomb aluminum panels covered by a highly diffusive coating from Labsphere with reflectivity above 
Table 2

Photometric/Spectroscopic Properties of 50 SNe Ia

\begin{tabular}{|c|c|c|c|c|c|c|c|}
\hline SN & $\begin{array}{l}\Delta m_{15}{ }^{\mathrm{a}} \\
(\mathrm{mag})\end{array}$ & $\begin{array}{l}\text { Opt. } \\
\text { Epochs }\end{array}$ & $\begin{array}{c}\text { NIR } \\
\text { Epochs }\end{array}$ & $\begin{array}{l}\text { Opt. Std. } \\
\text { Epochs }\end{array}$ & $\begin{array}{l}\text { NIR. Std. } \\
\text { Epochs }\end{array}$ & $\begin{array}{l}\text { Spectral } \\
\text { Subtype }\end{array}$ & $\begin{array}{c}T(B)_{\max } \\
\text { (days) }^{\mathrm{b}}\end{array}$ \\
\hline $2005 \mathrm{hj}$ & $0.787(003)$ & 17 & 12 & 4 & 5 & normal & $\ldots$ \\
\hline $2005 \mathrm{ku}$ & $1.019(060)$ & 7 & 4 & 3 & 3 & normal & -1 \\
\hline $2005 \mathrm{mc}$ & $1.533(005)$ & 17 & $\ldots$ & 3 & $\ldots$ & normal & $\ldots$ \\
\hline 2006bd & & 10 & 4 & 2 & 4 & 91bg-like & +7 \\
\hline $2006 \mathrm{br}$ & $1.160(034)$ & 9 & 5 & 3 & 3 & normal & 0 \\
\hline $2006 \mathrm{bt}$ & $0.979(017)$ & 17 & 5 & 4 & 3 & peculiar & $\ldots$ \\
\hline $2006 \mathrm{ef}$ & $1.326(015)$ & 10 & $\ldots$ & 3 & $\ldots$ & normal & +37 \\
\hline 2006ej & $1.344(012)$ & 13 & 3 & 3 & 5 & normal & +30 \\
\hline 2006et & $1.094(007)$ & 27 & 19 & 4 & 10 & normal & +9 \\
\hline $2006 \mathrm{ev}$ & $1.323(012)$ & 11 & 10 & 3 & 5 & normal & +15 \\
\hline $2006 \mathrm{fw}$ & $1.078(109)$ & 6 & $\ldots$ & 3 & $\ldots$ & normal & +2 \\
\hline $2006 \mathrm{gj}$ & $1.533(009)$ & 19 & 13 & 6 & 3 & normal & +4 \\
\hline $2006 \mathrm{hb}$ & ... & 26 & 10 & 7 & 6 & 86G-like & +6 \\
\hline $2006 h x$ & $1.151(039)$ & 9 & 8 & 3 & 4 & normal & -3 \\
\hline 2006 is & $1.113(015)$ & 27 & 9 & 5 & 9 & normal & +4 \\
\hline $2006 \mathrm{kf}$ & $1.479(009)$ & 21 & 17 & 4 & 7 & normal & +30 \\
\hline 2006lu & $0.908(009)$ & 27 & 6 & 5 & 2 & normal & +8 \\
\hline $2006 \mathrm{ob}$ & $1.479(013)$ & 13 & 12 & 3 & 6 & normal & +25 \\
\hline 2006 os & $1.019(014)$ & 13 & 10 & 6 & 5 & normal & -2 \\
\hline 2006 ot & $0.848(008)$ & 17 & 14 & 4 & 5 & peculiar & +17 \\
\hline $2007 \mathrm{~A}$ & $0.946(022)$ & 10 & 10 & 3 & 5 & normal & -1 \\
\hline $2007 \mathrm{~N}$ & $1.795(038)$ & 23 & 15 & 4 & 5 & 91bg-like & +12 \\
\hline $2007 \mathrm{~S}$ & $1.096(008)$ & 20 & 18 & 5 & 3 & 91T-like & -2 \\
\hline 2007af & $1.115(006)$ & 28 & 26 & 3 & 12 & normal & -11 \\
\hline 2007ai & $0.740(012)$ & 17 & 7 & 4 & 5 & 91T-like & -2 \\
\hline 2007 as & $1.217(010)$ & 19 & 11 & 3 & 5 & normal & -2 \\
\hline $2007 a x$ & $1.872(034)$ & 13 & 8 & 4 & 3 & 91bg-like & -2 \\
\hline $2007 \mathrm{ba}$ & $1.895(034)$ & 16 & 16 & 3 & 8 & 91bg-like & +6 \\
\hline $2007 b c$ & $1.207(011)$ & 10 & 12 & 5 & 6 & normal & +3 \\
\hline 2007bd & $1.236(010)$ & 14 & 13 & 3 & 4 & normal & -4 \\
\hline $2007 \mathrm{bm}$ & $1.192(012)$ & 10 & 10 & 5 & 3 & normal & -4 \\
\hline $2007 \mathrm{ca}$ & $1.097(008)$ & 12 & 10 & 3 & 3 & normal & -4 \\
\hline 2007hx & $0.946(029)$ & 13 & 9 & 3 & 3 & normal & $\ldots$ \\
\hline 2007 if & $\ldots$ & 18 & 8 & 5 & 3 & SC & 0 \\
\hline 2007jg & $1.175(014)$ & 20 & 9 & 5 & 5 & normal & +10 \\
\hline $2007 \mathrm{jh}$ & $1.857(128)$ & 14 & $\ldots$ & 5 & $\ldots$ & 86G-like & +14 \\
\hline 2007le & $1.097(005)$ & 27 & 17 & 5 & 10 & normal & -9 \\
\hline $2007 \mathrm{~mm}$ & $1.931(119)$ & 9 & $\ldots$ & 3 & $\ldots$ & 86G-like & $\ldots$ \\
\hline $2007 \mathrm{nq}$ & $1.471(010)$ & 25 & 19 & 9 & 12 & normal & -4 \\
\hline 2007on & $1.621(004)$ & 38 & 29 & 10 & 14 & normal & -4 \\
\hline $2008 \mathrm{C}$ & $1.113(008)$ & 22 & 18 & 5 & 6 & normal & +5 \\
\hline 2008R & $1.544(009)$ & 13 & 8 & 3 & 4 & normal & +4 \\
\hline $2008 b c$ & $0.930(006)$ & 33 & 12 & 6 & 3 & normal & -10 \\
\hline $2008 \mathrm{bq}$ & $0.919(012)$ & 16 & 4 & 3 & 6 & normal & 0 \\
\hline $2008 \mathrm{fp}$ & $0.915(006)$ & 28 & 22 & 3 & 4 & normal & -4 \\
\hline 2008gp & $1.067(004)$ & 20 & 11 & 3 & 8 & normal & -6 \\
\hline $2008 \mathrm{hv}$ & $1.253(006)$ & 25 & 18 & 8 & 5 & normal & -6 \\
\hline $2008 \mathrm{ia}$ & $1.323(012)$ & 15 & 16 & 4 & 3 & normal & -2 \\
\hline $2009 \mathrm{~F}$ & $1.977(025)$ & 15 & 10 & 5 & 4 & 91bg-like & -5 \\
\hline $2009 \mathrm{dc}$ & $0.759(011)$ & 14 & 10 & 3 & 4 & SC & -8 \\
\hline
\end{tabular}

Notes.

a Values of $\Delta m_{15}$ for the normal and SN 1991T-like objects and SN 2006ot were determined using the "max" model of SNooPy (see Stritzinger et al. 2010, Equation (5)). In the case of the low-luminosity SN 1991bg and SN 1986G-like objects, the possible super-Chandrasekhar mass object SN 2009dc, and the peculiar SN 2006bt, estimates of $\Delta m_{15}$ were obtained from direct spline fits to the $B$-band light curve. Uncertainties are given in parentheses in millimagnitude.

${ }^{\mathrm{b}}$ Epoch of the spectrum (with respect to time of $B$-band maximum) used to determine spectral subtype. If no epoch is given, the spectral classification was taken from the literature: SN 2005hj (Quimby et al. 2007), SN 2005mc (Deng et al. 2005), SN 2006bt (Foley et al. 2010), SN2007hx (Bassett et al. 2007b), and in the case of SN 2007mm from a combination of Bassett et al. (2007a) and our own analysis of an SDSS-II spectrum.

$95 \%$ from $3000 \AA$ to $16000 \AA$. From $16000 \AA$ to $22000 \AA$ the reflectivity hovers around $90 \%$, and beyond $22000-25000 \AA$ the reflectivity drops to $75 \%$.
Placed behind the secondary mirror of the telescope are four photodiodes with NIST traceable calibrations that measure the power of the light shining off the flat-field screen. The 
Table 3

Optical and Near-infrared Photometry of the Local Sequences in the Standard System

\begin{tabular}{|c|c|c|c|c|c|c|c|c|c|c|c|c|}
\hline STAR & $\alpha(2000)$ & $\delta(2000)$ & $\begin{array}{c}u^{\prime} \\
(\mathrm{mag})\end{array}$ & $\begin{array}{c}g^{\prime} \\
(\mathrm{mag})\end{array}$ & $\begin{array}{c}r^{\prime} \\
(\mathrm{mag})\end{array}$ & $\begin{array}{c}i^{\prime} \\
(\mathrm{mag})\end{array}$ & $\begin{array}{c}B \\
(\mathrm{mag})\end{array}$ & $\begin{array}{c}V \\
(\mathrm{mag})\end{array}$ & $\begin{array}{c}Y \\
(\mathrm{mag})\end{array}$ & $\begin{array}{c}J \\
(\mathrm{mag})\end{array}$ & $\begin{array}{c}H \\
(\mathrm{mag})\end{array}$ & $\begin{array}{c}K_{s} \\
(\mathrm{mag})\end{array}$ \\
\hline \multicolumn{13}{|c|}{ SN 2005hj } \\
\hline 01 & 01:26:51.61 & $-01: 16: 32.78$ & $17.153(011)$ & $15.821(009)$ & $15.345(009)$ & $15.197(009)$ & $16.184(008)$ & $15.522(007)$ & $14.540(007)$ & $14.291(007)$ & $13.958(008)$ & \\
\hline 02 & $01: 26: 47.87$ & $-01: 16: 36.35$ & $18.084(016)$ & $16.286(007)$ & $15.659(007)$ & $15.450(007)$ & $16.727(008)$ & $15.907(007)$ & $14.700(007)$ & $14.407(007)$ & $13.996(006)$ & \\
\hline 03 & $01: 26: 38.12$ & $-01: 12: 08.18$ & $19.757(064)$ & $17.068(007)$ & $15.730(009)$ & $14.990(009)$ & $17.752(012)$ & $16.315(008)$ & $\ldots$ & $\ldots$ & & \\
\hline 04 & $01: 26: 53.82$ & $-01: 11: 25.42$ & $20.096(101)$ & $17.370(009)$ & 15.987(011) & $14.521(011)$ & $18.211(015)$ & $16.568(017)$ & $12.906(008)$ & $12.418(008)$ & $11.870(008)$ & . . \\
\hline 05 & $01: 26: 55.70$ & $-01: 15: 04.82$ & $17.673(011)$ & $16.534(007)$ & $16.140(007)$ & $16.014(007)$ & $16.855(008)$ & $16.288(007)$ & 15.391(007) & $15.179(006)$ & $14.875(009)$ & \\
\hline 06 & $01: 26: 58.24$ & $-01: 14: 24.36$ & $20.624(124)$ & $17.836(008)$ & $16.699(007)$ & $16.265(007)$ & $18.446(013)$ & $17.205(007)$ & $15.319(006)$ & $14.915(007)$ & $14.314(006)$ & \\
\hline 07 & $01: 26: 51.35$ & $-01: 13: 09.98$ & $19.066(035)$ & $17.542(007)$ & $16.919(007)$ & $16.657(007)$ & 17.974(009) & $17.173(007)$ & $15.880(008)$ & $15.562(011)$ & $15.092(016)$ & \\
\hline 08 & $01: 26: 32.44$ & $-01: 13: 00.56$ & $19.821(072)$ & $17.985(008)$ & 17.296(009) & $17.038(007)$ & $18.436(010)$ & $17.563(013)$ & $\ldots$ & $\ldots$ & & \\
\hline 09 & $01: 26: 52.79$ & $-01: 16: 51.41$ & $20.643(124)$ & $18.574(008)$ & $17.694(007)$ & $17.360(016)$ & $19.083(014)$ & $18.072(011)$ & $16.474(011)$ & $16.136(018)$ & $15.588(016)$ & \\
\hline 10 & $01: 26: 38.81$ & $-01: 10: 59.43$ & $19.236(179)$ & $19.067(011)$ & $17.692(008)$ & $16.668(007)$ & $19.806(022)$ & $18.284(008)$ & $\ldots$ & $\ldots$ & & \\
\hline
\end{tabular}

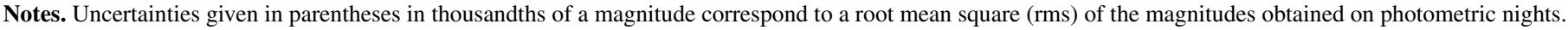

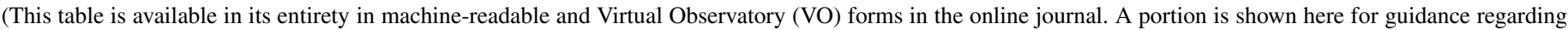
its form and content.)

photodiodes monitor the screen brightness and provide a signal proportional to the amount of light that enters the telescope. Two types of photodiodes are employed depending on the wavelength range being calibrated. Silicon photodiodes are used between $2500 \AA$ and $10000 \AA$, and germanium photodiodes best serve the purpose from $8000 \AA$ to $16500 \AA$. The signal from the photodiodes is amplified by a high-gain low-noise amplifier and read by an analog-to-digital converter acquisition system. The monochromator, lamps, and photodiodes are controlled remotely from the telescope control room. This setup is placed on the platform at the base of the telescope mount below the flat-field screen for the duration of the calibration run. Finally, from our own in lab tests we calibrated both the silicon and germanium photodiodes using the same NIST traceable standards and found that they both agree in their region of overlap.

\subsection{Calibration Procedure}

The calibration is made by comparing the number of photons measured by the photodiodes aimed at the flat-field screen to the number of counts measured during the same period by the science imager mounted on the telescope. It is a relative measurement. The acquisition system is automated; however, it is unable to communicate with the instrument control software, so part of the procedure is accomplished manually.

The optical bandpasses are scanned using a narrow bandwidth (FWHM $=26 \AA$ ) with a $20 \AA$ step between each measurement. Near-IR scans are performed with a fine step interval of $50 \AA$ and a coarser step interval of $200 \AA$. An image is taken at each interval step with a $30 \mathrm{~s}$ exposure time. Due to the low transmission sensitivity in the blue of the SITe3 chip in the direct-camera CCD, $120 \mathrm{~s}$ exposure times are required to accurately scan the $u$ band. To evaluate the repeatability of the calibration, each filter is scanned at least twice during nonsequential nights over the course of a calibration run.

\subsection{Calibration Results}

Two calibration runs were conducted at LCO during 2010. During the first of these runs (2010 January) the $u B g V r i$ bandpasses of the SITe3 CCD imager on the Swope telescope were measured twice per filter, point-to-point by wavelength, except for the $i$-band filter which was scanned only once. In a second run (2010 July) a second scan was obtained of the Swope

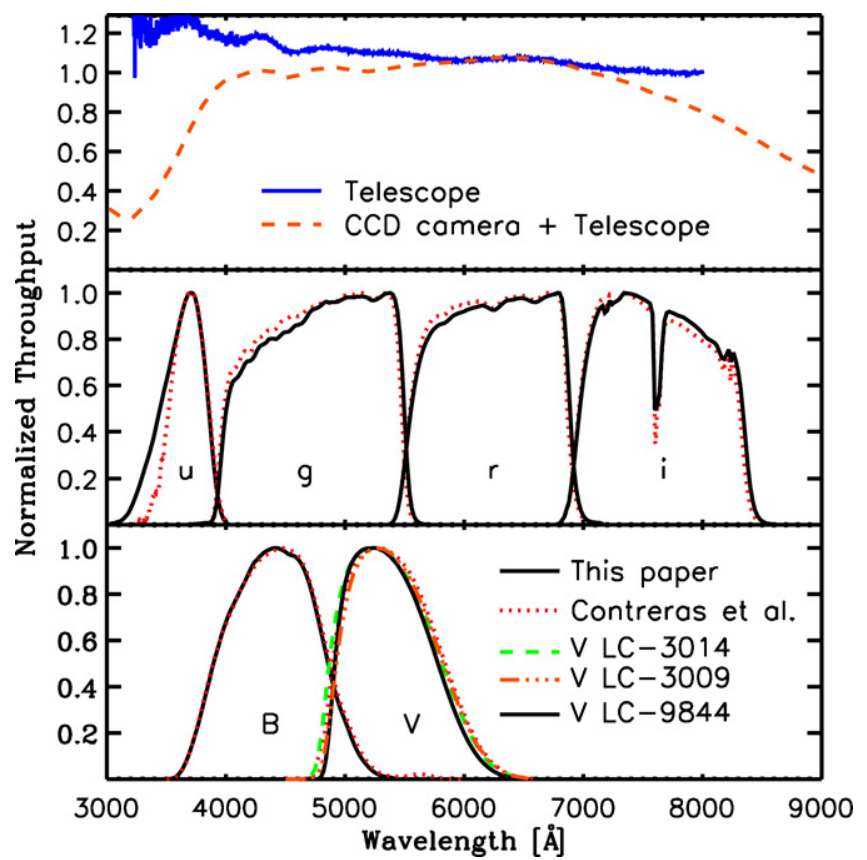

Figure 2. Results obtained from the photometric calibration of the Swope telescope equipped with CSP optical bandpasses. Top panel shows the throughput of the telescope with (dashed line) and without (solid line) the CCD camera. Both tracings have been normalized so that they cross at $6500 \AA$. Below are the scanned (solid lines) $u g r i$ and $B V$ bandpasses compared to those published by Contreras et al. (2010). Also included are the LC-3014 and LC-3009 $V$ bandpasses (see the text for details). To facilitate comparison the throughput of each bandpass has been normalized to unity. Note that all bandpasses have been multiplied by an atmospheric transmission function including telluric absorption features.

$i$ bandpass, and the near-IR bandpasses of both RetroCam on the Swope telescope and WIRC on the du Pont were scanned. The data from the second run are currently being analyzed and will be presented in a future publication. The repeatability of the $u B g V r$ measurements from the first calibration run was $\leqslant 1 \%$; by this we mean that there is a $\leqslant 1 \%$ point-to-point (by wavelength) uncertainty in the filter-response profiles. In other words, the ratios of the two scans of each individual filter fall within an envelope $\pm 1 \%$ wide.

Plotted in Figure 2 are the $u B g V r i$ bandpasses measured from our calibration program multiplied by a CTIO atmospheric 
transmission function, including telluric absorption features. Also included are the tracings previously published by Contreras et al. (2010, see their Figure 8). In addition to measuring the bandpasses, the response of the Swope telescope was measured with and without the SITe3 Direct CCD camera. The scanning without the CCD camera was performed with a photodiode placed at the focal plane of the telescope. These measurements yielded the optical throughput of the telescope which, when combined with the total throughput measurements, allowed the instrument response function $(\mathrm{CCD}+$ dewar window) to be deduced.

Under close inspection of the scanned bandpasses it was evident that in all cases the wings did not reach zero transmission. The cause of this effect was determined to be white light that leaked into the calibration setup. In general the offset produced by the white light was reasonably constant across the bandpasses; thus, to produce the final corrected curves the same constant value was subtracted from each bandpass trace.

As a direct result of our measurements, we managed to resolve two issues with the bandpasses published by Contreras et al. (2010). First, we now have an accurate measurement of the $u$ filter response function which, as discussed by Contreras et al. (2010), was a source of considerable uncertainty. Second, on 2006 January 14 UT the $V$ filter (called "LC-3014") was damaged and subsequently replaced with another $V$ filter ("LC3009"). After several nights of use, it was determined that the replacement filter had a significantly different color term compared to the original. This filter was therefore replaced with a third filter ("LC-9844") on 2006 January 25 UT, which was used for the remainder of the CSP campaigns. During the first photometric calibration run a portion of the broken LC-3014 filter and the temporary replacement, LC-3009, were also scanned using the monochromator while mounted on an optical bench. A comparison of these tracings indicates that the LC-9844 and LC-3014 V-band filters, though similar, are not identical (see Figure 2). Details regarding the color terms for these three $V$-band filters are provided in Appendix B.

\section{FINAL LIGHT CURVES}

Final optical and near-IR light curves of $50 \mathrm{SNe}$ Ia in the natural system are displayed in Figure 3. The corresponding optical and near-IR photometry of each object is provided in Tables 4 and 5, respectively. ${ }^{15,16}$ The quoted uncertainties in these tables correspond to the sum in quadrature of the instrumental error and the nightly zero-point error. In general, the light curves provide excellent temporal coverage and exhibit photometric precision at the 0.01-0.03 mag level.

The light curves of the "normal" and "SN 1991T-like" spectral subtypes (see Table 2) and SN 2006ot are overplotted in Figure 3 with light-curve fits (solid red lines) computed by SNooPy (Burns et al. 2011) using the "max model" (see Stritzinger et al. 2010, Equation (5)). From the SNooPy fits we obtain estimates of the peak magnitudes, the time of $B$-band maximum, $T\left(B_{\max }\right)$, the decline-rate parameter $\Delta m_{15}$ (Burns et al. 2011), and a covariance matrix of these parameters. Currently, SNooPy

\footnotetext{
15 Electronic files in ASCII format are available on the CSP Web site http://obs.carnegiescience.edu/CSP/data.

16 The small amount of optical photometry obtained with the du Pont telescope ( + Tek 5 CCD) is included in Table 4. These data, which are indicated with superscript "a" in the table, were reduced assuming that they are in the same natural system as the Swope photometry. This is a reasonable assumption since the exact same filters were used with both telescopes and with CCD detectors that had similar quantum efficiencies as a function of wavelength.
}

is unable to satisfactorily fit template light curves to peculiar SNe Ia like SN 2006bt (Foley et al. 2010), the fast-declining lowluminosity SN 1986G/SN 1991bg-like objects, or the possible super-Chandrasekhar mass SNe. In these cases, we interpolated the observed photometry with the use of spline functions, from which the peak magnitudes may be estimated, and the values of $T\left(B_{\max }\right)$ and $\Delta m_{15}$ were derived directly from the $B$-band spline fit. Overplotted on the observed photometry in Figure 3 are the resulting spline fits (red dashed lines). Due to insufficient photometric coverage, we were unable to accurately determine estimates of $\Delta m_{15}$ for the SN 1991bg-like objects SNe 2006bd and 2006hb, nor for the possible superChandrasekhar SN 2007if.

The $\Delta m_{15}$ values derived from either the SNooPy template light-curve fits or the spline fits are listed in Table 2, and are plotted in a histogram in Figure 4. We note that because a single value of $\Delta m_{15}$ is used in SNooPy to parameterize all the filters when performing the "max model" fit to an SN Ia, the values reported here can differ slightly from the value one would obtain by measuring it directly from the decline of just the $B$-band light curve. Also included in Figure 4 are the $\Delta m_{15}$ values for the $\mathrm{SNe}$ Ia presented in the first CSP data release of SNe Ia (Contreras et al. 2010).

We find that for the objects presented in this paper, 33 have pre-maximum optical coverage, and of these, 15 have observations beginning at least five days before $T\left(B_{\max }\right)$. Of the 45 SN Ia with near-IR observations, 27 have observations that commence prior to $T\left(B_{\max }\right)$, with a subset of 13 beginning at least five days earlier than maximum $B$-band light.

\section{SNe 2006bt AND 2006ot}

In a recent paper, Foley et al. (2010) called attention to one of the objects in our sample, SN 2006bt, which showed photometric and spectroscopic characteristics unlike those of any other known SN Ia. While possessing a broad, slowdeclining $\left(\Delta m_{15}=1.1\right) B$ light curve similar to a typical luminous SN Ia, SN 2006bt also displayed certain photometric and spectroscopic properties that are more similar to those of fast-declining, low-luminosity events. In particular, the $i$-band light curve showed only a weak secondary maximum, and the maximum and pre-maximum optical spectra displayed strong Si II $\lambda 5972$ absorption relative to Si II $\lambda 6355$ and a depression at $\sim 4100 \AA$ due to Ti II, all of which are properties characteristic of low-luminosity SN 1991bg-like objects. As mentioned earlier, our attempts to fit the light curves of SN 2006bt with SNooPy met with failure because of the combination of slow-declining light curves with a weak secondary maximum in the $i$ band. The $i$ light curve of SN 2006bt is also peculiar in reaching a primary maximum at essentially the same epoch as $B$ maximum, whereas for normal slow-declining $\mathrm{SNe}$ Ia, $i$ maximum occurs several days before $B$ maximum. Nevertheless, Foley et al. (2010) showed that the luminosity of SN 2006bt was quite typical of normal, slow-declining SNe Ia.

Interestingly, a second object in our sample, SN 2006ot, appears to be closely related to SN 2006bt. This is illustrated in Figure 5 where the $u B g V r i Y J H$ light curves of both $\mathrm{SNe}$ are overplotted. Also included in this figure are SNooPy fits to the CSP photometry of the normal, slow-declining $\left(\Delta m_{15}=1.0\right)$ SN 2006af (Contreras et al. 2010). Once again, in the observations of SN 2006ot we find the combination of broad, slowdeclining light curves with a weak secondary maximum in $i$. The morphology of the $Y$ and $J$ light curves of SN 2006ot is also peculiarly flat and consistent with the more limited CSP 

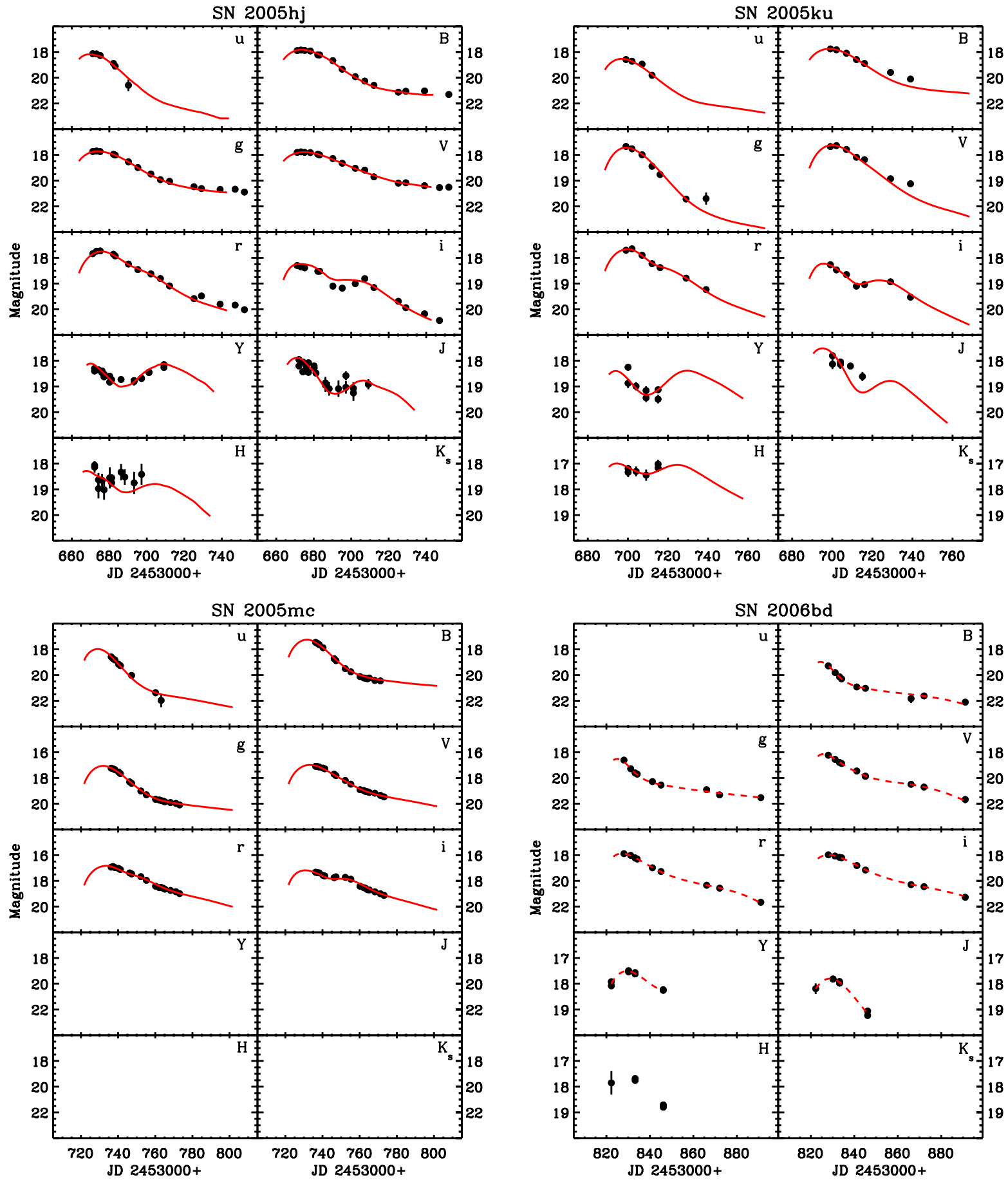

Figure 3. Optical $(\mathrm{uBg} V r i)$ and near-IR $\left(\mathrm{YJHK}_{s}\right)$ light curves of $50 \mathrm{SNe}$ Ia in the natural system of the Swope and du Pont telescopes. Uncertainties in the photometry are smaller than the points, unless shown. The smooth red curves correspond to the best template fits derived with SNooPy, while the dashed red curves correspond to spline fits (see Section 4 for details).

(A color and an extended version of this figure are available in the online journal.)

coverage obtained for SN 2006bt. Figure 6 shows a comparison of the spectra of SNe 2006bt and 2006ot near maximum light and approximately three to four weeks after maximum. Again, spectra of the normal SN 2006ax are included for comparison. The peculiar nature of the maximum-light spectrum of SN 2006bt compared to SN 2006ax is apparent in this figure, in particular the strength of the Si II $\lambda 5972$ absorption compared to $\lambda 6355$, and the broad Ti II absorption at $\sim 4100 \AA$. The maximumlight spectrum of SN 2006ot is different yet again, appearing in some ways intermediate between the spectra of SNe 2006bt and 2006ax (e.g., in the ratio of the equivalent widths of the Si II $\lambda 5972$ and $\lambda 6355$ features). Most striking is the strength, width, and blueshift of the Si II $\lambda 6355$ line, the minimum of which yields an expansion velocity of $\sim 14500 \mathrm{~km} \mathrm{~s}^{-1}$. At three to four weeks past maximum, the spectra of SNe 2006bt and 2006 ot closely resemble each other, while the spectrum of the normal SN 2006ax differs significantly at wavelengths shortward of $6000 \AA$. 
Table 4

Optical Photometry of 50 SNe Ia in the Swope Natural System

\begin{tabular}{|c|c|c|c|c|c|c|}
\hline $\begin{array}{l}\text { JD } \\
2453000+\end{array}$ & $\begin{array}{c}u \\
(\mathrm{mag})\end{array}$ & $\begin{array}{c}g \\
(\mathrm{mag})\end{array}$ & $\begin{array}{c}r \\
(\mathrm{mag})\end{array}$ & $\begin{array}{c}i \\
(\mathrm{mag})\end{array}$ & $\begin{array}{c}B \\
(\mathrm{mag})\end{array}$ & $\begin{array}{c}V \\
(\mathrm{mag})\end{array}$ \\
\hline \multicolumn{7}{|c|}{ SN 2005hj } \\
\hline 671.66 & $18.137(025)$ & $17.737(008)$ & $17.839(010)$ & $18.300(014)$ & $17.890(008)$ & $17.804(008)$ \\
\hline 673.64 & $18.157(025)$ & $17.696(008)$ & $17.743(012)$ & $18.351(014)$ & $17.849(010)$ & $17.764(008)$ \\
\hline 675.62 & $18.274(024)$ & $17.743(010)$ & $17.734(014)$ & $18.390(016)$ & $17.879(008)$ & $17.802(014)$ \\
\hline $678.70^{\mathrm{a}}$ & $\ldots$ & $\ldots$ & $\ldots$ & $\ldots$ & $17.920(008)$ & $17.820(008)$ \\
\hline 683.64 & $19.104(043)$ & $18.014(014)$ & $17.928(014)$ & $18.521(021)$ & $18.227(012)$ & $18.000(012)$ \\
\hline 690.67 & $20.586(452)$ & $18.536(028)$ & $18.242(025)$ & $19.102(040)$ & $18.671(048)$ & $18.285(032)$ \\
\hline 695.69 & $\ldots$ & $18.982(016)$ & $18.458(017)$ & $19.179(029)$ & $19.339(024)$ & $18.650(014)$ \\
\hline 702.65 & $\ldots$ & $19.483(021)$ & $18.624(016)$ & $19.005(021)$ & $19.916(026)$ & $19.043(017)$ \\
\hline 707.71 & $\ldots$ & $19.925(035)$ & $18.806(021)$ & $18.810(033)$ & $20.261(073)$ & $19.181(028)$ \\
\hline
\end{tabular}

Notes. Uncertainties given in parentheses in thousandths of a magnitude.

a Images obtained with the du Pont telescope.

(This table is available in its entirety in machine-readable and Virtual Observatory (VO) forms in the online journal. A portion is shown here for guidance regarding its form and content.)

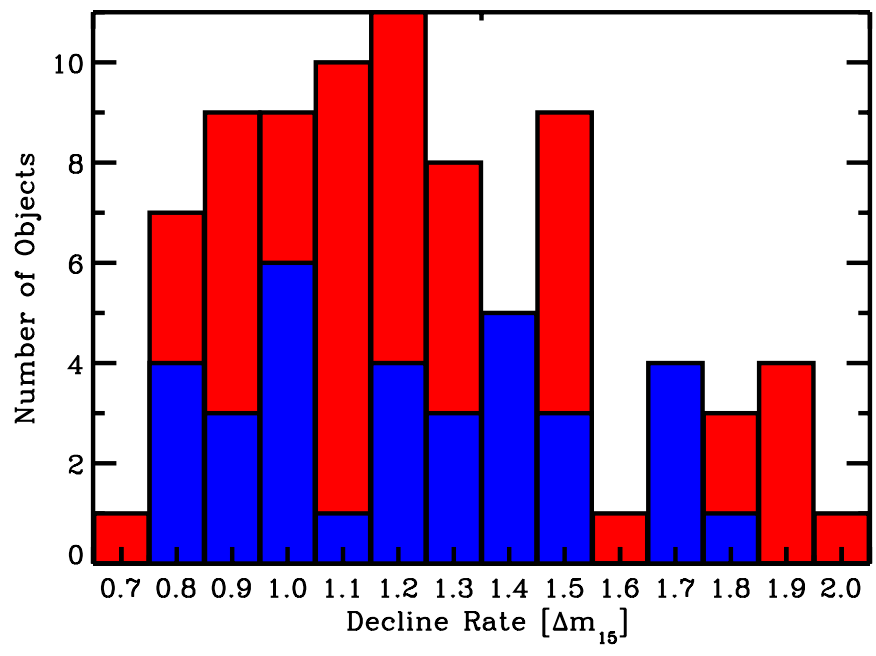

Figure 4. Stacked bar chart of the $\Delta m_{15}$ values for 47 of the $50 \mathrm{SNe}$ Ia presented in this paper (red) and 34 of the 35 objects (blue) from the first CSP SN Ia data release (Contreras et al. 2010). The $\Delta m_{15}$ values were computed with the "max model," or via spline fits in the case of the 11 non-standard objects (see Table 2). The current CSP sample covers the full range of decline rates, with the distribution peaking between $\Delta m_{15}=1.1$ and 1.2.

According to the maps of Schlegel et al. (1998), interstellar reddening of $\mathrm{SNe} 2006 \mathrm{bt}$ and 2006ot due to dust in the Milky Way was small $\left(A_{V}=0.17 \mathrm{mag}\right.$ and $A_{V}=0.05 \mathrm{mag}$, respectively). Foley et al. (2010) did not find evidence for Na I D absorption in the spectrum of SN 2006ot at the redshift of its host galaxy, nor is $\mathrm{Na}$ I $\mathrm{D}$ detectable in our maximum-light spectrum of SN 2006ot. Hence, it is unlikely that the light of either SN suffered large dust extinction. The difference in host galaxy recession velocities corrected to the cosmic microwave background $(\mathrm{CMB})$ reference frame $\left(v_{\mathrm{CMB}}=9737 \mathrm{~km} \mathrm{~s}^{-1}\right.$ for $\mathrm{SN} 2006 \mathrm{bt}$ and $v_{\mathrm{CMB}}=15695 \mathrm{~km} \mathrm{~s}^{-1}$ for $\mathrm{SN} 2006 \mathrm{ot}$ ) implies a brightness difference of $\sim 1.0 \mathrm{mag}$ if both objects were unreddened and shared the same peak luminosity. This is only $0.1 \mathrm{mag}$ different from the shift of $\sim 0.9$ mag required to register the light curves of both $\mathrm{SNe}$ (see Figure 5). Thus, the peak luminosities were likely quite similar. Interestingly, the host of SN 2006bt was an S0/a galaxy and NED lists the morphology of the host of SN 2006ot as Sa, implying a relatively old progenitor (Foley et al. 2010).

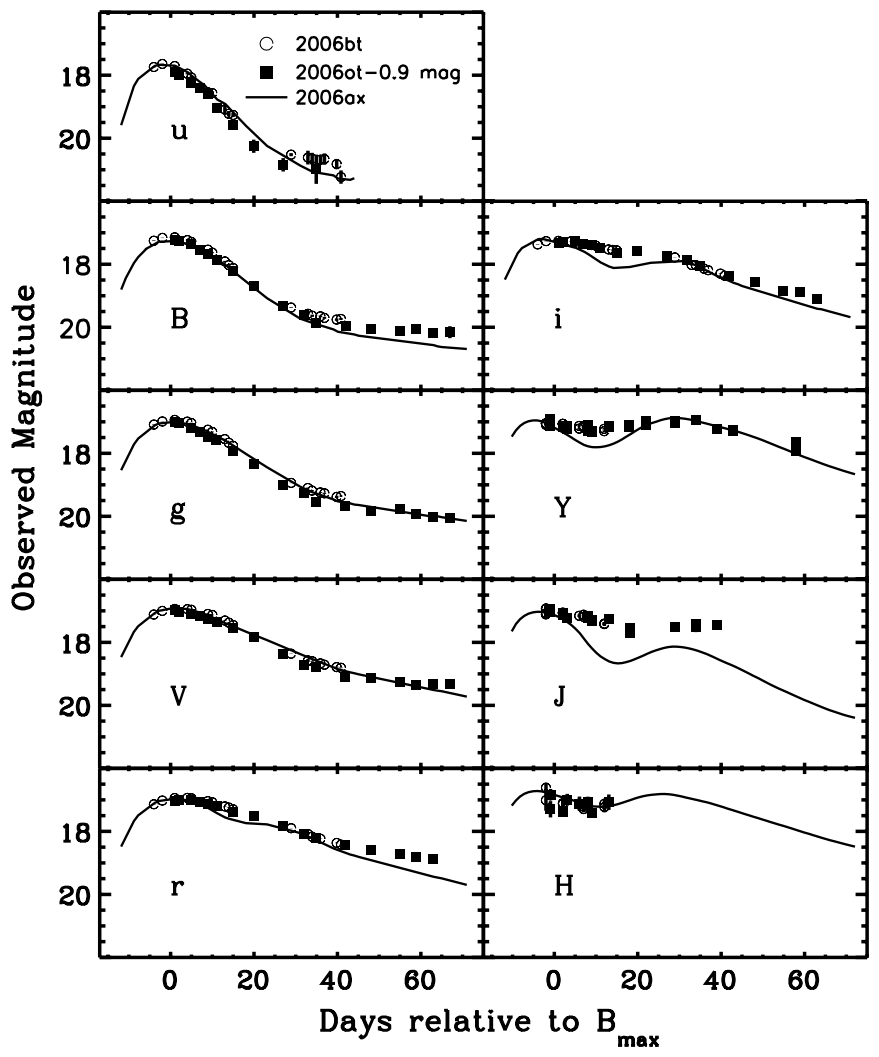

Figure 5. Comparison of light curves of the normal Type Ia SN 2006ax (solid line) to those of the peculiar SNe 2006bt (circles) and 2006ot (filled squares).

It is not at all obvious how SNe 2006bt and 2006ot fit into the overall scheme of SNe Ia, but it is clear that they can easily be confused with normal events unless high-quality photometry and/or spectroscopy is obtained. This is particularly true at high redshifts where rest system $i$ or near-IR light curves (where the photometric differences are greatest) are almost never obtained. Foley et al. (2010) concluded that contamination of SNe Ia samples by similar objects will both increase the scatter of the Hubble diagram and systematically bias measurements of cosmological parameters. Fortunately, they do not appear to be very common in the local universe as only two such events 
Table 5

Near-infrared Photometry of $45 \mathrm{SNe}$ Ia

\begin{tabular}{lccccc}
\hline \hline JD & $Y$ & $J$ & $H$ & $K_{s}$ & Telescope $^{\mathrm{a}}$ \\
$2453000+$ & $(\mathrm{mag})$ & $(\mathrm{mag})$ & $(\mathrm{mag})$ & $(\mathrm{mag})$ & \\
\hline 672.60 & $18.349(072)$ & $18.081(095)$ & $18.102(180)$ & $\ldots$ & SWO \\
674.65 & $18.357(060)$ & $18.239(097)$ & $18.801(301)$ & $\ldots$ & SWO \\
676.60 & $18.432(077)$ & $18.322(119)$ & $18.653(258)$ & $\ldots$ & SWO \\
677.66 & $18.575(076)$ & $18.265(101)$ & $19.016(387)$ & $\ldots$ & SWO \\
680.69 & $18.716(102)$ & $18.241(129)$ & $18.553(407)$ & $\ldots$ & SWO \\
681.70 & $18.736(073)$ & $18.482(070)$ & $18.650(142)$ & $\ldots$ & DUP \\
686.73 & $18.731(111)$ & $18.908(251)$ & $18.334(318)$ & $\ldots$ & SWO \\
688.71 & $\ldots$ & $19.090(267)$ & $18.521(303)$ & $\ldots$ & SWO \\
693.68 & $18.791(137)$ & $19.088(322)$ & $18.750(428)$ & $\ldots$ & SWO \\
697.63 & $18.685(110)$ & $18.809(195)$ & $18.418(408)$ & $\ldots$ & SWO \\
701.65 & $18.452(088)$ & $19.165(266)$ & $\ldots$ & $\ldots$ & SWO \\
709.62 & $18.195(058)$ & $18.913(167)$ & $\ldots$ & $\ldots$ & SWO \\
\hline
\end{tabular}

Notes. Uncertainties given in parentheses in thousandths of a magnitude.

${ }^{a}$ DUP and SWO correspond to the du Pont and Swope telescopes, respectively.

(This table is available in its entirety in machine-readable and Virtual Observatory (VO) forms in the online journal. A portion is shown here for guidance regarding its form and content.)

are among the $87 \mathrm{SNe}$ Ia with photometry published to date by the CSP.

\section{CONCLUSION AND PROSPECTS FOR THE FUTURE}

We have presented the second data release of SN Ia photometry obtained by the CSP. Combined with the first sample (Contreras et al. 2010) and photometry from Schweizer et al. (2008) and Stritzinger et al. (2010), the CSP data set currently consists of 87 objects. The high-quality and dense temporal coverage, extending over nine filters from the optical to the near-IR, provides a sample that is well suited for tackling the issues related to accurately estimating host-galaxy reddening (e.g., Folatelli et al. 2010).

With the addition of the 50 low $-z$ objects presented in this paper, we are in a position to extend upon our first analysis and, among other things, to provide a detailed investigation of the fastest declining SNe Ia. The CSP sample now consists of 16 rapid decliners $\left(\Delta m_{15}>1.5\right)$. This sample is nearly evenly split between the morphologies of the near-IR maximum of those objects that peak in the near-IR before $T\left(B_{\max }\right)$, and those that peak after. Krisciunas et al. (2009) suggest that objects whose near-IR light curves peak after $T\left(B_{\max }\right)$ appear to be $0.5-0.8 \mathrm{mag}$ less luminous in the optical and near-IR than those events whose near-IR light curves peak before $T\left(B_{\max }\right)$. It will be particularly interesting to re-examine this issue using the CSP sample, as well as to compare other properties of the light curves and spectra of this SN Ia subtype.

When completely reduced and analyzed, the CSP optical and near-IR light curves of SNe Ia already in hand will constitute a fundamental reference for cosmological studies. However, at present, the full utility of the CSP sample as a reference for observations at high redshift is compromised by the fact that the median redshift of the sample is $\sim 0.02$. At this redshift, the rootmean-square (rms) error in distance modulus due to peculiar velocities is $\pm 0.13 \mathrm{mag}$ or $6 \%$ in distance and dominates the observed Hubble diagram dispersion (Neill et al. 2007; Folatelli et al. 2010). This problem can be minimized by extending observations to SNe further out in the smooth Hubble flow. We have therefore proposed and been granted NSF funding to carry

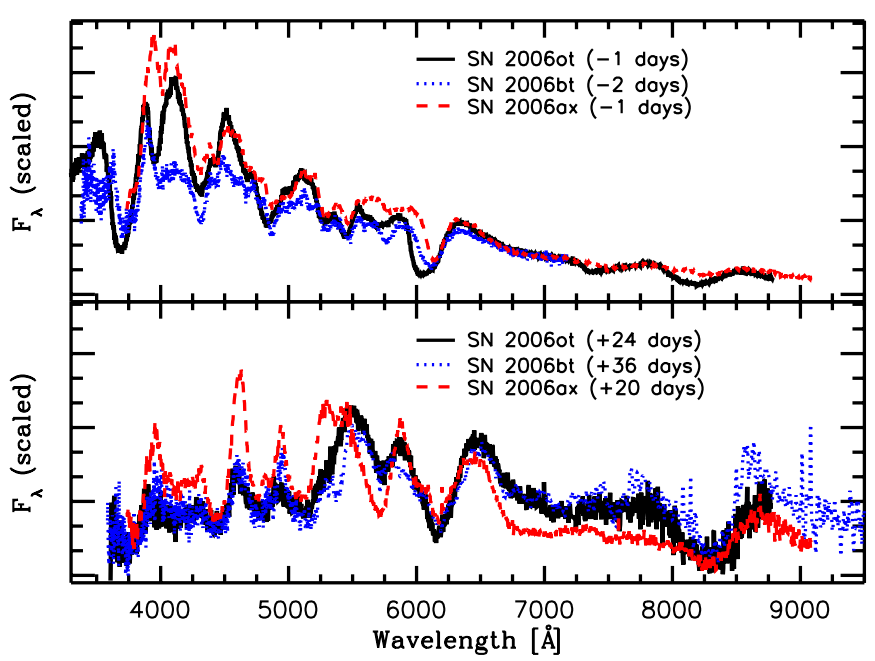

Figure 6. Spectral comparisons of the normal Type Ia SN 2006ax (red dashed line) to those of the peculiar SNe 2006ot (black solid line) and 2006bt (blue dotted line). The spectra of SN 2006bt are reproduced from Foley et al. (2010), and the -1 day spectrum of SN 2006ot is from J. Silverman et al. 2011 (in preparation). All other spectra were obtained by the CSP, and will be published in G. Folatelli et al. (2012, in preparation).

(A color version of this figure is available in the online journal.)

out a second stage of the CSP with the goal of producing optical and near-IR light curves of 100-200 SNe Ia in the redshift range $0.03<z<0.08$. With such a sample we expect the rms error due to peculiar motions to drop to $\pm 3 \%$ or less in distance.

The CSP extends special thanks to the mountain staff of the Las Campanas Observatory for their assistance throughout the duration of our observational program, and to Jim Hughes for his superb support of our network of computers. This material is based upon work supported by the NSF under grants AST-0306969, AST-0908886, AST-0607438, and AST1008343. The Dark Cosmology Centre is funded by the Danish National Research Foundation. M.H. acknowledges support by CONICYT through grants FONDECYT Regular 1060808, Centro de Astrofisica FONDAP 15010003, Centro BASAL CATA (PFB-06), and by the Millennium Center for Supernova Science (P06-045-F). A.V.F. is grateful for the financial support of the NSF and the TABASGO Foundation. This research has made use of the NASA/IPAC Extragalactic Database (NED) which is operated by the Jet Propulsion Laboratory, California Institute of Technology, under contract with the National Aeronautics and Space Administration.

\section{APPENDIX A}

\section{PHOTOMETRIC ZERO POINTS}

With the scanned system-response functions presented in Section 3.3, we proceed to compute photometric zero points following the prescription described by Contreras et al. (2010, see their Appendix C).

The photometric zero point for bandpass $X$, with a response function denoted by $S_{X}(\lambda)$, can be expressed as

$$
\mathrm{ZP}_{X}=2.5 \log \left[\int_{0}^{\infty} S_{X}(\lambda) F_{o}(\lambda) \frac{\lambda}{h c} d \lambda\right]+m_{0}
$$

Here $F_{o}(\lambda)$ is the spectral energy distribution (SED) of a fiducial source and $m_{o}$ is the observed magnitude of this source through the bandpass $X$. 
Table 6

Optical Color Terms for CSP Bandpasses in the Landolt System

\begin{tabular}{lcc}
\hline \hline Filter & Method1 & Method2 \\
\hline$u$ & $+0.050(002)$ & $+0.049(027)$ \\
$g$ & $-0.014(001)$ & $-0.015(013)$ \\
$r$ & $-0.016(001)$ & $-0.016(016)$ \\
$i$ & $+0.000(001)$ & $+0.000(015)$ \\
$B$ & $+0.069(001)$ & $+0.069(013)$ \\
$V$ LC-3014 & $-0.059(002)$ & $-0.060(015)$ \\
$V$ LC-3009 & $-0.034(006)$ & $-0.034(009)$ \\
$V$ LC-9844 & $-0.063(001)$ & $-0.065(010)$ \\
\hline
\end{tabular}

Note. Uncertainties given in parentheses in thousandths of a magnitude.

To compute the $B$ - and $V$-band zero points we adopt the SED of Vega ( $\alpha$ Lyr) as our fiducial source. In doing so, we use the Bohlin \& Gilliland (2004) SED of Vega (version 5) obtained from CALSPEC, ${ }^{17}$ and the Fukugita et al. (1996) standard magnitudes $B_{\text {std }}=0.03$ and $V_{\text {std }}=0.03$. As we aim to compute zero points in the natural system of the Swope telescope, $B_{\text {std }}$ and $V_{\text {std }}$ are transformed to the Swope natural system using the following equations:

$$
\begin{gathered}
B_{\text {nat }}=B_{\text {std }}-\mathrm{CT}_{B} \times\left(B_{\text {std }}-V_{\text {std }}\right), \\
V_{\text {nat }}=V_{\text {std }}-\mathrm{CT}_{V} \times\left(V_{\text {std }}-i_{\text {std }}\right),
\end{gathered}
$$

where $\mathrm{CT}_{X}$ are the color terms. Color terms for the CSP bandpasses were given by Hamuy et al. (2006) and Contreras et al. (2010); the former values correspond to the average for the first of five yearly observing campaigns, while the slightly different numbers given in the latter reference are based on the first four observing campaigns. In Table 6, the final fiveyear average color terms are presented. The second column corresponds to the unweighted average after applying a $5 \sigma$ clipping algorithm. Listed in the third column as a consistency check are the median values calculated without the $5 \sigma$ clipping, with uncertainties corresponding to 1.49 multiplied by the median absolute deviation. As is seen, the differences between these two methods of computation are minimal. We therefore adopt the unweighted averages determined with the $5 \sigma$ clipping.

In Equation (A3), the value of " $i_{\text {std }}$ " was computed synthetically using the SED of Vega and the "USNO 40" $i^{\prime}$ bandpass. This yields $i_{\text {std }}^{\prime}=0.382 \mathrm{mag}$. Executing the transformations yields Vega magnitudes in the Swope natural system of $B_{\text {nat }}=0.03$ and $V_{\text {nat }}=0.009$.

Armed with $B_{\text {nat }}$ and $V_{\text {nat }}$, and the $B$ and $V$ (LC-9844) scanned bandpasses, zero points were computed directly using Equation (A1). In addition, zero points for the temporary (LC3009) and replacement (LC-3014) $V$-band filters discussed in Section 3.3 were also computed, and the results are listed in Table 7.

We note that due to the limited use of the temporary LC$3009 V$ filter, we are unable to accurately determine the color term of this filter from observations of standard-star fields. To remedy this problem, the color term was estimated synthetically using an atlas of Landolt (1992) spectrophotometic standards (Stritzinger et al. 2005), and the corresponding known $B_{\text {std }}$ and $V_{\text {std }}$ magnitudes (see Appendix B for further details).

To compute the zero points of the scanned ugri bandpasses, $\mathrm{BD}+17^{\circ} 4708$ was chosen as the fiducial source. Similar to the

\footnotetext{
$17 \mathrm{ftp}: / / \mathrm{ftp}$. stsci.edu/cdbs/current_calspec/alpha_lyr_stis_005.ascii.
}

Table 7

Photometric Zero Points of CSP Optical Bandpasses

\begin{tabular}{lc}
\hline \hline Filter & Zero Point \\
\hline$u$ & 13.044 \\
$g$ & 15.135 \\
$r$ & 14.915 \\
$i$ & 14.781 \\
$B$ & 14.344 \\
$V$ LC-3014 & 14.540 \\
$V$ LC-3009 & 14.493 \\
$V$ LC-9844 & 14.450 \\
\hline
\end{tabular}

steps described above, the standard $u_{\mathrm{std}}=10.56, g_{\mathrm{std}}=9.64$, $r_{\text {std }}=9.35$, and $i_{\text {std }}=9.25 \mathrm{mag}$ of $\mathrm{BD}+17^{\circ} 4708$ (Fukugita et al. 1996) were transformed to the Swope natural system using the color term in Table 6 and the following transformation equations:

$$
\begin{gathered}
u_{\text {nat }}=u_{\text {std }}-\mathrm{CT}_{u} \times\left(u_{\mathrm{std}}-g_{\mathrm{std}}\right), \\
g_{\text {nat }}=g_{\mathrm{std}}-\mathrm{CT}_{g} \times\left(g_{\mathrm{std}}-r_{\mathrm{std}}\right), \\
r_{\text {nat }}=r_{\mathrm{std}}-\mathrm{CT}_{r} \times\left(r_{\mathrm{std}}-i_{\mathrm{std}}\right), \\
i_{\text {nat }}=i_{\mathrm{std}}-\mathrm{CT}_{i} \times\left(r_{\mathrm{std}}-i_{\mathrm{std}}\right) .
\end{gathered}
$$

These transformed magnitudes were then compared to synthetic magnitudes computed with our scanned bandpasses and the Bohlin \& Gilliland (2004) SED of BD $+17^{\circ} 4708$ to compute the zero points via Equation (A1); the results are listed in Table 7.

\section{APPENDIX B}

\section{$B$ - AND $V$-BAND COLOR TERMS}

To test the accuracy of the scanned $B$ and $V$ bandpasses, "synthetic" color terms were computed by comparing synthetic photometry derived using the Stritzinger et al. (2005) set of Landolt (1992) spectrophotometric standards to the published Landolt values. If the scanned bandpasses are correct, the synthetic color terms derived from this experiment should match the observed values reported in Table 6.

Plotted in the left panel of Figure 7 are the differences between the synthetic $B$-band magnitudes computed with the scanned bandpass and the published Landolt values versus synthetic $B-V$ color. Applying a color cut to exclude stars bluer than $B-V=0$ or redder than $B-V=0.9$ mag yields the best-fit color term of $\mathrm{CT}_{B}=+0.065$. This is reasonably consistent with the value of $\mathrm{CT}_{B}=+0.069$ given in Table 6 .

Presented in the right panel of Figure 7 is a similar comparison for the three $V$ bandpasses used by the CSP. The blue points correspond to values derived with the scan of the broken LC-3014 filter, while the red points are from the scan of the temporary LC-3009 filter, and the green points are the values obtained with the scan of the replacement filter LC-9844. Adopting the previously mentioned color cut yields best-fit color terms of $\mathrm{CT}_{V}=-0.063, \mathrm{CT}_{V}=-0.044$, and $\mathrm{CT}_{V}=$ -0.065 for the LC-3014, LC-3009, and LC-9844 bandpasses, respectively. These values are in excellent agreement with the color terms derived from the broadband photometry of the Landolt standard-star observations (see Table 6). 

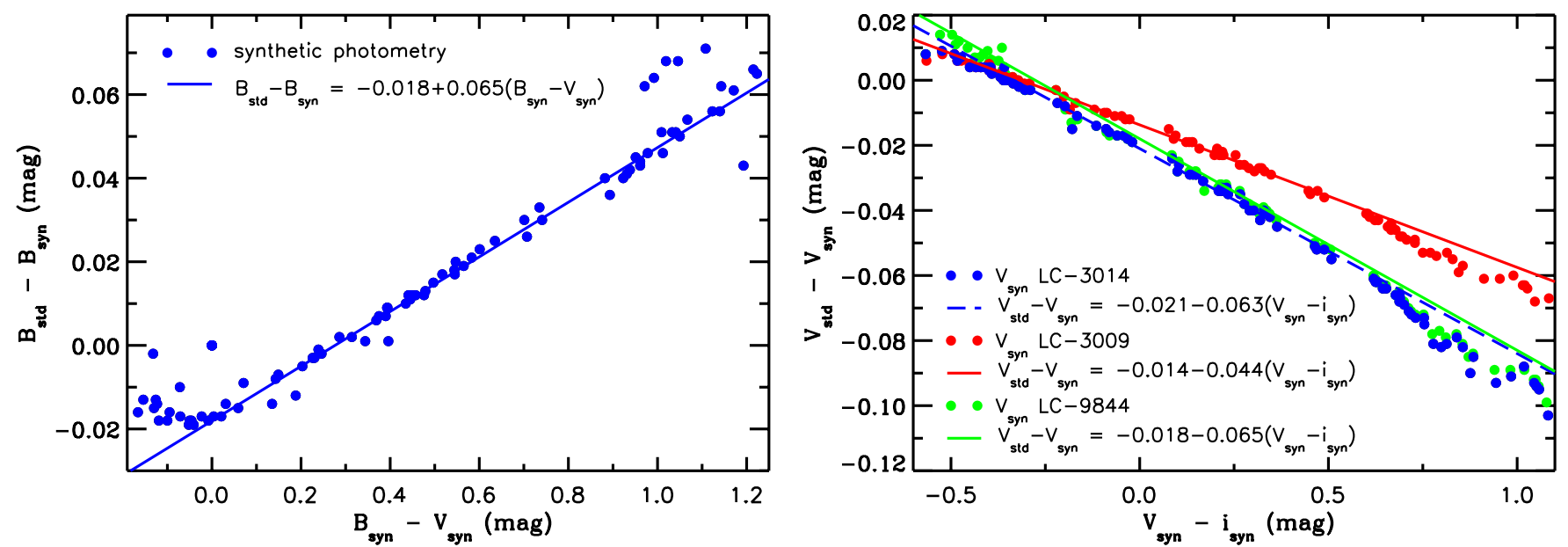

Figure 7. Derivations of $B$ - and $V$-band color terms derived synthetically from the scanned bandpasses and the Stritzinger et al. (2005) atlas of Landolt spectrophotometric standards. The best linear fits are indicated with lines. Prior to determining the best linear fits, a color cut was applied to exclude those stars with $B-V$ values bluer than 0 and redder than 0.9 mag. This left a subsample of 44 standards in which the best fits were determined.

The results of this experiment confirm the accuracy of the scanned $B$ and $V$ bandpasses. Unfortunately, due to a lack of spectrophotometric measurements of the Smith et al. (2002) standard stars, it is not possible to accurately apply this test to the scanned ugri bandpasses.

\section{APPENDIX C}

\section{$V$-BAND LC-3009 PHOTOMETRY}

As the $V$-band LC-3009 color term has a non-negligible difference with respect to the two other $V$-band filters used, we re-examined the small amount of photometry obtained with LC3009. During the time the LC-3009 filter was used, 12 different SNe Ia were observed during one to three epochs. Nine of these objects are included in Contreras et al. (2010). For completeness we returned to these nights in question and recomputed the photometry in the natural system of the Swope telescope. We note that for these objects, eliminating the observations taken with the LC-3009 filter has little effect on the quality or coverage of the $V$-band light curves, except for the case of SN 2006D whose first three pre-maximum epochs were obtained with LC3009. Updated $V$-band photometry for these three epochs is available on the CSP Web site.

\section{REFERENCES}

Allen, S., Rapetti, D. A., Schmidt, R. W., et al. 2007, MNRAS, 383, 879 Astier, P., Guy, J., Regnault, N., et al. 2006, A\&A, 447, 31

Bassett, B., Becker, A., Bizyaev, D., et al. 2007a, CBET, 1102, 1 Bassett, B., Becker, A., Brewington, H., et al. 2007b, CBET, 1057, 1 Blondin, S., \& Tonry, J. L. 2007, ApJ, 666, 1024

Bohlin, R. C., \& Gilliland, R. L. 2004, AJ, 127, 3508

Burns, C., Stritzinger, M., Phillips, M. M., et al. 2011, AJ, 141, 19

Contreras, C., Hamuy, M., Phillips, M. M., et al. 2010, AJ, 139, 519

Deng, J., Kawabata, K. S., Hattori, T., et al. 2005, CBET, 334, 1

Dunkley, J., Komatsu, E., Nolta, M. R., et al. 2009, ApJS, 180, 306

Filippenko, A. V. 2005, in ASP Conf. Ser. 332, The Fate of the Most Massive Stars, ed. R. Humphreys \& K. Stanek (San Francisco, CA: ASP), 33
Filippenko, A. V., Li, W. D., Treffers, R. R., \& Modjaz, M. 2001, in ASP Conf. Ser. 246, Small-Telescope Astronomy on Global Scales, ed. W. P. Chen, C. Lemme, \& B. Paczyński (San Francisco, CA: ASP), 121

Filippenko, A. V., Richmond, M. W., Branch, D., et al. 1992a, AJ, 104, 1543

Filippenko, A. V., Richmond, M. W., Matheson, T., et al. 1992b, ApJ, 384, L15

Folatelli, G., Phillips, M. M., Burns, C. R., et al. 2010, AJ, 139, 120

Foley, R., Narayan, G., Challis, P. J., et al. 2010, ApJ, 708, 1748

Freedman, W. L., Burns, C. R., Phillips, M. M., et al. 2009, ApJ, 704, 1036

Frieman, J. A., Bassett, B., Becker, A., et al. 2008, AJ, 135, 338

Fukugita, M., Ichikawa, T., Gunn, J. E., et al. 1996, AJ, 111, 1748

Ganeshalingam, M., Li, W., Filippenko, A. V., et al. 2010, ApJS, 190, 418

Giannantonio, T., Scranton, R., Crittenden, R. G., et al. 2008, Phys. Rev. D, 77, 12350

Hamuy, M., Folatelli, G., Morrell, N. I., et al. 2006, PASP, 118, 2

Hicken, M., Challis, P., Jha, S., et al. 2009, ApJ, 700, 331

Howell, D. A., Sullivan, M., Nugent, P. E., et al. 2006, Nature, 443, 308

Krisciunas, K., Marion, G. H., Suntzeff, N. B., et al. 2009, AJ, 138, 1584

Landolt, A. U. 1992, AJ, 104, 340

Leibundgut, B., et al. 1993, AJ, 105, 301

Neill, J. D., Hudson, M. J., \& Conley, A. 2007, ApJ, 661, L123

Perlmutter, S., et al. 1999, ApJ, 517, 565

Persson, S. E., Murphy, D. C., Krzeminski, W., Roth, M., \& Rieke, M. J. 1998, AJ, 116, 2475

Phillips, M. M. 1993, ApJ, 413, L105

Phillips, M. M., Phillips, A. C., Heathcote, S. R., et al. 1987, PASP, 99, 592

Phillips, M. M., Wells, L. A., Suntzeff, N. B., et al. 1992, AJ, 103, 1632 Quimby, R. M. 2006, PhD thesis, Univ. Texas

Quimby, R. M., Höflich, P., \& Wheeler, J. C. 2007, ApJ, 666, 1083

Rheault, J.-P., et al. 2010, Proc. SPIE, 7735, 773564

Riess, A. G., Filippenko, A. V., Challis, P., et al. 1998, AJ, 116, 1009

Riess, A. G., Strolger, L.-G., Casertano, S., et al. 2007, ApJ, 659, 98

Scalzo, R. A., Aldering, G., Antilogus, P., et al. 2010, ApJ, 713, 1073

Schlegel, D. J., Finkbeiner, D. P., \& Davis, M. 1998, ApJ, 500, 525

Schweizer, F., Burns, C. R., Madore, B. F., et al. 2008, AJ, 136, 1482

Smith, J. A., Tucker, D. L., Kent, S., et al. 2002, AJ, 123, 2121

Stritzinger, M., Burns, C. R., Phillips, M. M., et al. 2010, AJ, 140, 2036

Stritzinger, M., Hamuy, M., Suntzeff, N. B., et al. 2002, AJ, 124, 2100

Stritzinger, M., Suntzeff, N. B., Hamuy, M., et al. 2005, PASP, 117, 810

Sullivan, M., et al. 2011, ApJ, in press, arXiv:1104.1444

Williams, A. J. 1997, PASA, 14, 208

Wood-Vasey, W. M., Friedman, A. S., Bloom, J. S., et al. 2008, ApJ, 689, 377

Wood-Vasey, W. M., Miknaitis, G., Stubbs, C. W., et al. 2007, ApJ, 666, 694 